\title{
A Novel Member of the Ig Superfamily, turtle, is a CNS-Specific Protein Required for Coordinated Motor Control
}

\author{
Kale D. Bodily, Clayton M. Morrison, Robert B. Renden, and Kendal Broadie \\ Department of Biology, University of Utah, Salt Lake City, Utah 84112-0840
}

We describe here the cloning and functional characterization of a neural-specific novel member of the Ig superfamily, turtle (tutl), with a structure of five Ig C2-type domains, two fibronectin type III domains, and one transmembrane region. Alternative splicing of the tutl gene produces at least four Tutl isoforms, including two transmembrane proteins and two secreted proteins, with primary structures closely related to a human brain protein (KIAA1355), the Deleted in Colorectal Cancer/Neogenin/Frazzled receptor family, and the Roundabout/Dutt1 receptor family. An allelic series of tutl gene mutations resulted in recessive lethality to semilethality, indicating that the gene is essential. In contrast to other family members, tutl does not play a detectable role in axon pathfinding or nervous system morphogenesis. Likewise, basal synaptic transmission and locomotory movement are unaffected. However, tutl mutations cause striking movement defects exhibited in specific types of highly coordinated behavior. Specifically, tutl mutants display an abnormal response to tactile stimulation, the inability to regain an upright position from an inverted position (hence, "turtle"), and the inability to fly in adulthood. These phenotypes demonstrate that tutl plays an essential role in establishing a nervous system capable of executing coordinated motor output in complex behaviors.

Key words: Drosophila; Ig superfamily; coordination; motor control; behavior; cell adhesion; motor neuropathy
An integrated nervous system requires the establishment of complex neural connections that are made possible through elaborate signaling and adhesion pathways involving cell-surface, extracellular matrix, and chemotrophic molecules as attractive or repulsive guidance cues (Tessier-Lavigne, 1994; Goodman, 1996; Dickson, 1998; Cooper et al., 1999; Wright et al., 1999). One subfamily of the Ig superfamily (IgSF) is a conserved class of cell-surface receptors that mediate axon guidance and target recognition in vertebrate and invertebrate systems. Examples include the Drosophila/grasshopper Fasciclin adhesion molecules (Harrelson and Goodman, 1988; Wright et al., 1999), the Drosophila/mouse Roundabout (Robo)/Dutt1 receptors (Kidd et al., 1998; Sundaresan et al., 1998), the mammalian/Drosophila Neogenin (Neo)/ Deleted in Colorectal Cancer (DCC)/Frazzled (Fra) receptors (Kolodziej et al., 1996; Cooper et al., 1999), and the vertebrate neural cell adhesion molecule/L1/Axonin adhesion molecules (Barthels et al., 1987; Kohl et al., 1992; Kunz et al., 1998).

IgSF members function in neural development in many different ways. Some function as cell-surface chemorepellant or chemoattractant receptors, such as the Robo/Dutt1 (Kidd et al., 1998; Sundaresan et al., 1998) and Neo/DCC/Fra (Keino-Masu et al., 1996; Kolodziej et al., 1996; Cooper et al., 1999) proteins,

\footnotetext{
Received Nov. 27, 2000; revised Jan. 29, 2001; accepted Feb. 22, 2001.

This work was supported by National Institute of Health Grant GM54544 and by a Muscular Dystrophy Association grant to K.B. K.D.B. received support from the Bioscience Undergraduate Research Program, the Undergraduate Research Opportunities Program at the University of Utah, and the Arnold and Mable Beckman Scholars Fellowship. We thank Guy Tear for advice and Fasciclin II antibody (mAb 1D4), Kelly Beumer for assistance with immunohistochemistry, Emma Rushton for assistance with genetics, and Michael Bastiani for assistance with and use of his confocal microscope. We are grateful to the Bloomington Drosophila Stock Center for fly stocks and to the Berkeley Drosophila EST project led by Gerry Rubin for generating and distributing the EST clones used in this study.

Correspondence should be addressed to Dr. Kendal S. Broadie, Department of Biology, University of Utah, 257 South 1400 East, Salt Lake City, UT 84112-0840. E-mail: broadie@biology.utah.edu.

Copyright (C) 2001 Society for Neuroscience 0270-6474/01/213113-13\$15.00/0
}

respectively. Others function as cell-surface adhesion molecules in homophilic or heterophilic interactions, such as the Fasciclin proteins (Harrelson et al., 1988). In addition, other family members exist as both membrane-bound and secreted forms, such as the Axonin-1 proteins (Stoeckli et al., 1991). The functional repertoire of this extensive family is further increased through alternative splicing of primary transcripts (Schmucker et al., 2000) and by post-translational modifications such as glycosylation (Huang et al., 1997).

Analysis of the Drosophila genome sequence reveals a total of $153 \mathrm{Ig}$ domain proteins and 46 proteins containing fibronectin (Fn) domains (Adams et al., 2000). It is probable that only a subset of these genes play roles in nervous system development and/or function, yet genomic analysis suggests that numerous important neural receptors have not been characterized. In Caenorhabditis elegans, 19 of the total $64 \mathrm{Ig}$ domain proteins appear to function in neural development (defined by similarity to characterized homologs), a surprising number considering the extreme simplicity of the nervous system of the nematode (Teichmann and Chothia, 2000). In light of the potential size and diversity of the neural IgSF, coupled with the complexity of nervous system development, it is likely that numerous IgSF members play roles in neural development and function via novel mechanisms.

In this study, we report the cloning and functional characterization of Drosophila turtle (tutl), a novel member of the IgSF that is expressed in the CNS and in a small, defined subset of the peripheral nervous system (PNS). Alternative splicing of the primary transcript results in membrane-bound and secreted Tutl isoforms that are essential for development and adult viability. tutl function is required for establishing a nervous system capable of executing complex forms of coordinated movement such as tactile escape response, coordinated righting behavior in larval and adult stages, and flight in adulthood. The characteristic 
inability of a tutl mutant to turn over when flipped on its back gave rise to the gene name.

\section{MATERIALS AND METHODS}

Genomic analysis. A genomic nucleotide sequence from P1 and bacterial artificial chromosome (BAC) clones obtained from the Berkeley Drosophila Genome Project (BDGP) database (www.fruitfly.org) was analyzed using Computational Genomics Group nucleotide analysis software (http://genomic.sanger.ac.uk/gf/gfb.html). Gene structure analysis was performed using BLASTP software available at the National Center for Biotechnology Information (NCBI) Advanced Blast Search website (http://www.ncbi.nlm.nih.gov/blast/blast.cgi?Jform = 1). A genomic sequence containing the predicted gene locus was compared with the expression sequence tag (EST) sequence also using the NCBI Advanced Blast Search. Four cDNA clots $(7979,14288,6591$, and 6419) were identified on the BDGP database (www.fruitfly.org/cgi-bin/bfd/ clonereport.pl) from EST nucleotide sequence homology to the region of the predicted gene.

Gene structure analysis. All available cDNA clones from clots 7979, 14288, 6591, and 6419 were obtained and sequenced. Sequence data were compared with the genomic sequence to identify intron-exon structure as well as to predict alternative splicing in the gene. Nucleotide sequence data were used to predict the amino acid sequence of protein products for each of the four identified Tutl isoforms as well as the longest possible derived isoform. The protein sequence was analyzed for functional domain structure using InterProScan software (www.ebi.ac.uk/interpro/ interproscan/ipsearch.html). Transmembrane (TM) domains were predicted using Swiss Institute for Experimental Cancer Research Tmpred software (www.ch.embnet.org/software/TMPRED form.html).

$m R N A$ expression studies. Digoxygenin-labeled DNA probes were made by PCR using the a PCR DIG Probe synthesis kit (catalog no. 1636 090; Boehringer Mannheim, Indianapolis, IN). Using a cDNA template (clone I.D. number GH16705), primers 5'-ATACAGGTGCTCCAGTTCGT- $3^{\prime}$ and 5' $^{\prime}$-TCCTCTGGCGTAACACTAAA- $3^{\prime}$ as well as digoxygenin-11-dUTP, a $451 \mathrm{nt}$ probe complimentary to a region of exon 3-4, were synthesized. Whole-mount in situ hybridization was conducted on Drosophila embryos according to the method of Tautz and Pfeifle (1989). Probes against the AP-50 mRNA were used as a positive control (Zhang and Broadie, 1999).

Mutagenesis and mutant characterization. A BLAST search identified a complementation group in the predicted gene region containing P-element inserts 1(2)01085 and 1(2)k14703 as well as the 24E deficiency (Df(2L)ed-dp). The 1(2)01085, 1(2)k14703, and Df(2L)ed-dp stocks were obtained from the Bloomington Stock Center (Bloomington, IN). The location of P-element insert 1(2)01085 was identified to be within tutl exon 4 at the 24E1-E2 region. P-element insert 1(2)k14703 was localized by PCR to the genomic region spanning between the $3^{\prime}$ border of exon 8 and the $5^{\prime}$ border of exon 11 using primers 5'-CCAAAAACCCCGCCCCTGTACCTAT-3' and ' ' $^{\prime}$-ACAAAGCGTCCATCGAGGCTCCGTT-3' as well as Herculase Enhanced DNA Polymerase (Stratagene, La Jolla, CA), confirming that both inserts localized to the coding region of tutl.

A P-element mobilization strategy was used to generate deletions that remove portions of the tutl gene. We mobilized l(2)k14703 via transient introduction of transposase $(\Delta 2-3)$ and created stocks of each excision event balanced over the Curly of Oster $(\mathrm{CyO})$ balancer chromosome (Oster, 1956). Lethal lines that failed to complement the 24E deficiency (Df(2L)ed-dp) comprised a single complementation group: turtle. Four of these deletions (tutl1, tutl2, tutl3, and tutl4) were analyzed by PCR. DNA from homozygous animals was extracted using a Puregene DNA isolation kit (catalog \#D 5000A; Gentra Systems, Minneapolis, MN) and used in PCRs with the following primer sets to map chromosomal end points for each deficiency (Df): 1A, 5'-GAAATCCGTAGAGTCCAAAG3'; 1B, 5'-GATCCTTTGGGAAACTGGAA-3'; 2A, 5'-CCACGTAAAAGTCAACGGTT-3'; 2B, 5'-CGCAACCGCACTTATTTACA-3'; 3A, 5'-ATACAGGTGCTCCAGTTCGT-3'; 3B, 5'-CAAGAGGCCTAAACAAAAGC-3'; 4A, 5'-GCGACTCATAAACCGAATCA-3'; 4B, 5' TCCTCTGGCGTAACACTAAA-3'; 5A, 5'-ATCCCATTTTCCCATACCCA-3'; 5B, 5'-CCCTGGTGTTCCTCATTGAC-3'; 6A, 5'-TCTTTCAGCTAATGCCAGAC-3'; 6B, 5'-GGTTTGCAATGTGCTTTCGA-3'; 7A, 5'-GTACCTACATTCCGAGTTTC-3'; 7B， 5'-CGGGTTAAAGAGAAACTCAG-3'; 8A, 5'-CATTCTCATTGCCATACTCC-3'; 8B, 5' GCCATACAGATTATTGCTGC-3'; 9A, 5'-CATTCAGTAGCGCTTCTCCA-3'; 9B, 5'-GTAGCAGTTGAAGACCACGT-3'

Genetic rescues of the 1(2)k14703 tutl mutation were achieved by mobilization of the P-element as described to generate precise excision events. Precise excision was identified by rescue of the semilethal phenotype exhibited by $1(2) \mathrm{k} 14703$ mutants. We conducted all behavioral assays described in this paper on homozygous revertant animals and found that the precise excision of the $1(2) \mathrm{k} 14703$ P-element rescued all behavioral phenotypes exhibited by tutl mutants (the escape response, the larval roll-over response, the adult roll-over response, and flight capability).

Behavioral assays. Canton-S (CS) larvae were used as controls in all assays. Mutant tutl chromosomes were balanced over the $\mathrm{CyO}$ [ubiquitin green fluorescent protein (GFP)] balancer chromosome to establish stocks containing an in vivo chromosome marker. All heterozygous tutl mutants were identified by the presence of GFP expression, and all homozygous tutl mutants used in the behavioral assays were identified by the absence of GFP expression. Embryos were selected from $3 \mathrm{hr}$ laying periods on agar plates. Genotyped embryos were transferred to fresh agar plates containing equal portions of live yeast in aliquots of 15-25 embryos per plate within $12 \mathrm{hr}$ of laying time. Embryo aliquots were then raised at $25^{\circ} \mathrm{C}$ in a temperature- and humidity-controlled incubator. All larval assays were conducted on L3 larvae 90-102 hr after fertilization. All adult assays were conducted on flies 12-24 hr after eclosion.

The locomotion assay in L3 larvae was conducted by placing a single larva on a fresh, room temperature agar plate. Three peristaltic contraction wave measurements were recorded for each animal and then averaged to produce one data point. The escape response was elicited by cephalic tactile stimulation using a 25 gauge hypodermic needle. Touch stimulation was made at the anteriormost portion of the animal holding the needle in-line with the anteroposterior axis of the larva. The larval response was observed for 5-15 sec after stimulation.

The L3 larval roll-over assay was conducted on isolated individuals on a fresh, room temperature agar plate. Using forceps, the animals were rolled over to an inverted position as defined by the ventral midline. On release of the animal, a timer was started; the timer was stopped when the animal had completely righted itself, as defined by the dorsal midline being completely vertical. Three roll-over time measurements were recorded for each animal and then averaged to produce one data point.

The adult roll-over assay was conducted on individuals at room temperature on a standard laboratory work bench. Because of their inability to fly, tutl 1 , tutl 2 , and $1(2) \mathrm{k} 14703$ flies were easily contained in an open environment. CS and revertant flies, however, had to be caught within a glass tube and handled individually. Using forceps, the flies were flipped over on their backs, and a timer set to $60 \mathrm{sec}$ was started. Five groups of 10 adults were assayed individually in this manner, and the percentage of adults that could right in $60 \mathrm{sec}$ was calculated. All statistical analyses (Mann-Whitney $U$ tests) were performed using Instat software (Graph Pad, San Diego, CA).

Histology, immunocytochemistry, and confocal microscopy. All immunocytochemistry and histological staining was performed on tutl mutant stocks balanced over the CyO [fushi tarazu LacZ] balancer for genotyping purposes. Embryonic studies were conducted on staged embryos raised at $25^{\circ} \mathrm{C}$, fixed for $20 \mathrm{~min}$ in $4 \%$ paraformaldehyde in PBS $(0.02 \mathrm{M}$ phosphate buffer and $0.1 \mathrm{M} \mathrm{NaCl}, \mathrm{pH} 7$ ), and then washed in $0.1 \%$ Triton $\mathrm{X}-100$ in PBS (PBS-TX) several times over a period of $1 \mathrm{hr}$. Wholemount preparations were incubated overnight at $4^{\circ} \mathrm{C}$ with rabbit monoclonal antisera raised against $\beta$-galactosidase $(\beta$-gal) in PBS $(1: 1000$; Cappel, Durham, NC) and mouse monoclonal antibody (mAb) BP102 (1:500; developed by C. S. Goodman, University of California Berkeley, Berkeley, CA, obtained from the Developmental Studies Hybridomae Bank at the University of Iowa, Iowa City, Iowa), with mAb ID4 against Fasciclin II (FasII) (1:5; developed by C. S. Goodman, obtained from G. Tear, King's College, London, UK), or with mAb 22C10 against Futsch (1:500; developed by S. Benzer, California Institute of Technology, Pasadena, CA, obtained from the Developmental Studies Hybridoma Bank at the University of Iowa).

Larval immunocytochemical studies were conducted on L3 larvae $\left(90-102 \mathrm{hr}, 25^{\circ} \mathrm{C}\right)$ reared in normalized conditions as described for behavioral assays. Larvae were dissected along the dorsal midline, fixed for $45 \mathrm{~min}$ in $4 \%$ paraformaldehyde, and washed as described above. Preparations were incubated overnight at $4^{\circ} \mathrm{C}$ with anti-synaptotagmin (1:500; Littleton et al., 1993) and mAb ID4 (1:5, gift from G. Tear) as described above.

Adult fly heads from 2- to 7-d-old animals $\left(25^{\circ} \mathrm{C}\right)$ were fixed and mounted in paraffin wax; next, $7 \mu \mathrm{m}$ histological slices were made through the head according to Protocol 112 in Ashburner (1989). Preparations were washed in PBS-TX with BSA several times over a period of $1 \mathrm{hr}$. Preparations were incubated overnight at $4^{\circ} \mathrm{C}$ with rabbit monoclo- 


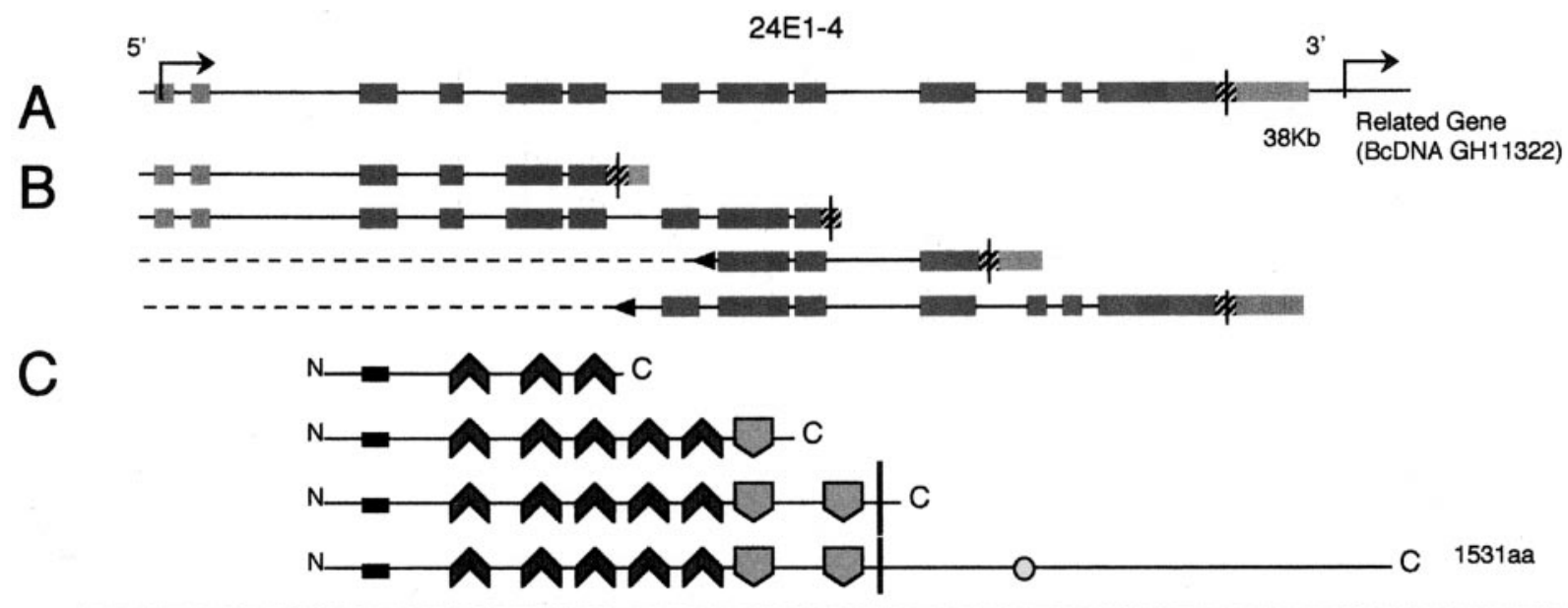

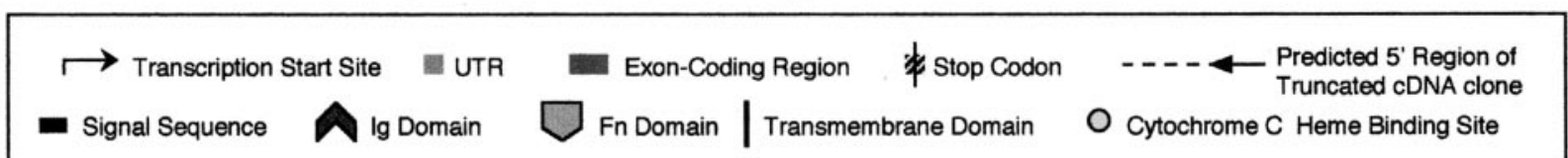

D MGVCADLGSH RWCRALSTQH NTEKSKEQQQ QSQPLEIPEQ RASKCRGDID RTTTTTIPAS KTLTASPAKT AAFTVKTTRR RRSRRRAEGS SICVPIRRGQ GSTPTPTIQV LQFVLVSLLA LLAKNAQAHN IPEDAVHITA ILGEGVIENC HVEEPNDHPV PYVLOWDKKV SETGSDLPIY IWYESYPEHI EEGYKGRVSR VSODSPFGSA SLNLTNIRES DOGWYECKVV FLNRDPKQHK NGTWFHLDVH APPRFSVTPE DIIYVNLGDS IILNCOADGT PTPBILWYKD ANPVDPSPTV GIFNDGTELR ISTIRHEDIG EYTCIARNGE GQVSHTARVI IAGGAVIMVP PTNQTKLBGE KVIFSCBAKA MPGNVTVRWY REGSPVREVA ALETRVTIRK DGSLIINPIK PDDSGQYLCE VTNGIGDPQS ASAYLSVEYP AKVTFTPTVQ YLPFRLAGVV OCYIKSSPOL OYVTWTKDKR LLEPYOMKDI VVMANGSLLF TRVNEEHOGO YACTPYNAQG TAGASGVMDV LVRKPPAFTV EPETLYQRKV GDSVEMHCDA LEABGTERPT IKWOROEGEO LIESORNRIK ISGGNITIEN LRREDFGYYQ CVVSNEVATL MAVTQLVIBG TQPHAPYNIT GKATESSITL QWLPGYSGGS EYKQDYTIWF REAGVNDWQT ISVTPSGSTQ VTINGLASGT TYEFQVVGRN VLGDGMMSKV MTVRTLEDAP AAPRNVKAAT QPPDSFFQLM PDEAGPKPGP PRAVSVTEVX NGFLITWQSP LERAHIVKFY TIKYRTDAQW KTLNRGQIRP EETQYLVKNL VGGRTYYFRV LANSEKSYES SDEVKFPVPA RVKHKAITAG VVGGILFFIV AIILSVCAVK ICNKRKRRKQ BKEFNMVACR ITDARNIAAN NHHLHNRSTG SISSGQVPLK NNTEHYRDYE SVFIVPGPSC HNQNRYKQTR ISSLTAILIA ILHWIWPPDR CtnChSIYSS PNLEDGDEDD GGGGRRRSVS RIQRSLDGRF VLDVEGVSKL GYSQQTLESG NVDVVDGGLF ERRNSNVSQK SSSDDGGFLS RRNFITARAS WRRPLVASSS QLSLQSAADS ARGFLQGLLK IGAKQSAVPP NSQSYFDEAV SGARYQNVLR PYTSSNNLYG NADRSRPLHI NTISGSLSQQ QQLYTPSISR IFSSSPQQLQ PHHQQLLLSS GGSGAYPTHF SDLSTVYPPN SAERSSHNLS SRYRYYSQEL PSLRTIQEET RRQQQQKQHP LBDHFVPLQL PSPPSWRSYY QSQASYRPRT RWYPRHHSRL FSNRQQQHEM LSPLPQLNLN LRNSMNPGGL BASPESRSSS SGFGSKNTSN HPGSTSEWRL LPPYRAPPSP PKHSGYFGGQ QAQGQTPHGS YSYPRATTPP YTMAHWLEMI SRLNAATDSN LPKAPCPVDV GSVDGHYBFD PATPTPSASS MLREDLNLHI DTHPYHHHTL GPLSGSLLHS HAPYGGGARK QRSLPAHLPR YDNVEVRLQA MREEFYAYRK RQAMQQMESV C

Figure 1. turtle gene structure and its predicted protein product. A, Genomic intron-exon structure of the tutl region. A $40 \mathrm{~kb}$ genomic fragment is depicted showing the structure of the tutl gene including exons, introns, stop codons, and untranslated transcribed regions. Note that the transcription start site of the BcDNA GH11322 gene is at the $3^{\prime}$ end of the genomic diagram. B, Splice variants of the tutl gene. One full-length cDNA was identified (GH15753) and partial-length clones (GH16705, GH08133, and LD28224/LP03459) identify three other distinct splice variants of the tutl gene, each demonstrating unique $3^{\prime}$ structures. $C$, Protein structure for the Tutl isoforms including type and location of functional domains and transmembrane regions. $D$, Amino acid sequence of the Tutl protein. Italic text indicates the putative signal sequence (first) and the putative transmembrane region (second). Underlined text indicates Ig domains. Bold text indicates Fn domains. Lowercase text indicates a cytochrome c heme binding site.

nal antisera raised against $\beta$-galactosidase in PBS (1:1000; Cappel) and mouse mAb BP102 (1:500; Developmental Studies Hybridoma Bank) as described above.

All preparations were incubated in either Alexa Red anti-rabbit or Alexa Green anti-mouse secondary antibodies (1:500; Vector Laboratories, Burlingame, $\mathrm{CA}$ ) for $2 \mathrm{hr}$ at $25^{\circ} \mathrm{C}$ and washed as described above. Preparations were mounted in glycerol, and fluorescent images were acquired on a Radiance 2000 confocal microscope (Bio-Rad, Hercules, CA). Images were presented using Adobe Photoshop 5.0 and Claris Draw 1.0.2 software.

Electrophysiology: two-electrode voltage-clamp. Two-electrode voltageclamp (TEVC) recordings were performed on muscle 6 in anterior abdominal segments (A2-A4) of L3 larva, according to previously published methods (Rohrbough et al., 2000). Briefly, dissected larvae were placed in a Plexiglas recording chamber and viewed in transmitted light using a compound microscope (Zeiss, Thornwood, NY) fitted with Differential Interference Contrast (Nomarski) optics and a $40 \times$ waterimmersion lens. Recordings were made at $18^{\circ} \mathrm{C}$ with sharp glass electrodes pulled from fiber-filled borosilicate glass (World Precision Instruments, Sarasota, FL) to resistances of $10-25 \mathrm{M} \Omega$ and filled with a solution of a $3: 1$ mixture of $3 \mathrm{M} \mathrm{KAc} / \mathrm{KCl}$. Stimulation of the motor nerve was achieved by brief $(0.5-0.8 \mathrm{msec})$ positive current stimulation of a loop of motor nerve in a suction electrode, using a Grass S88 Simulator (Grass Instruments, Warwick, RI). The nerve was stimulated at $0.5 \mathrm{~Hz}$. Suction electrodes were made using pulled electrodes of fiber-filled borosilicate glass that were heat-polished to final inner diameter of 10-12 $\mu \mathrm{m}$ and filled with bath saline. Recording bath solution was a modified standard saline, consisting of (in $\mathrm{mm}$ ): $128 \mathrm{NaCl}, 2 \mathrm{KCl}, 4 \mathrm{MgCl}, 70$ sucrose, and 5 HEPES. $\mathrm{CaCl}$ was added to solutions with a $\mathrm{pH}$ of 7.2 , to bring the final $\left[\mathrm{Ca}^{2+}\right]$ to $0.4 \mathrm{~mm}$.

Current recordings were from voltage-clamped $(-60 \mathrm{mV})$ cells using standard voltage-clamp techniques and an Axoclamp 2B patch-clamp amplifier (Axon Instruments, Foster City, CA). The signal was filtered at $0.5 \mathrm{kHz}$ online, converted to a digital signal using a DigiData 1200 analog-to-digital interface (Axon Instruments), and stored on computer (Gateway P5-166 mHz) for later analysis. All analysis was done offline, using the pClamp6 program suite (Axon Instruments). Forty responses were recorded per larva and then averaged to give each data point.

\section{RESULTS}

The turtle gene encodes a novel member of the Ig superfamily

We designed a systematic analysis of sequenced P1 and BAC clones beginning on the left arm of Drosophila chromosome 2 in an attempt to locate genes essential for neural development. In this search, we identified a hypothetical gene in the 24E1-24E3 cytological region that appeared to encode a novel IgSF protein (Fig. 1C). We identified five distinct EST clots in the genomic 


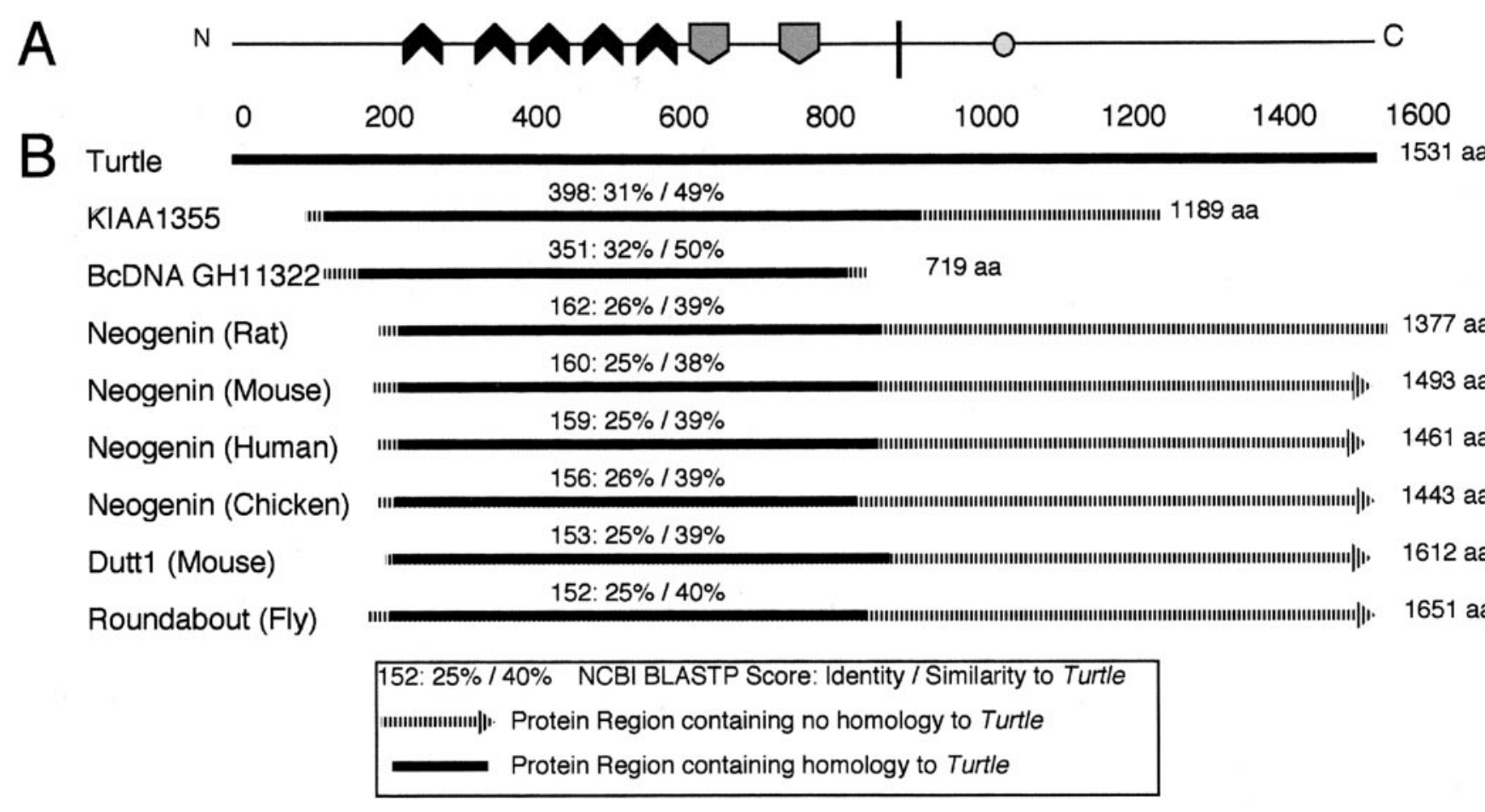

Figure 2. Closest known relatives of the Tutl protein. $A$, The structure of the Tutl protein (see Fig. 1 for symbol legend). $B$, Similarity between the Tutl protein and its closest known relatives as defined by amino acid sequence similarity. KIAA1355 is a predicted human protein expressed in the fetal brain. BcDNA GH11322 is a relative of the tutl gene located adjacent to the tutl locus. Neogenin is a conserved, cell-surface chemoattractant receptor that functions in axon guidance in the CNS. Roundabout is a conserved, cell-surface chemorepellant receptor that functions in axon guidance in the CNS. Dutt1 is the mouse homolog of Roundabout.

region containing the predicted gene (Fig. $1 B$ ), suggesting the possibility of five separate transcriptional units. Annotation of the recently compiled Drosophila genome (www.fruitfly.org/annot/ bands/band24.html) suggested the presence of three distinct transcriptional units (CG15426, CG15427, and CG15427). We obtained all available cDNA clones and completely sequenced each clone. Analysis of sequence data indicated that the predicted genes CG15426, CG15427, and CG15427 are in fact portions of a single transcription unit (Fig. $1 A$ ). The predicted gene product is an integral membrane protein that is 1531 amino acids (aa) in size with an extracellular domain that contains a possible signal sequence for cell-surface localization, five Ig C2 type repeats, two Fn type III repeats, a TM region, and a sizable intracellular domain containing a cytochrome $\mathrm{C}$ heme-type binding site (Fig. $1 C$ ). Additional analysis of cDNA and the genomic nucleotide sequence revealed that alternative splicing produces at least four protein isoforms, which represent two transmembrane and two secreted proteins (Fig. $1 B, C$ ). We designated this gene turtle based on its distinctive mutant behavioral phenotype (see below).

Sequence analysis of the primary structure of the tutl protein showed that it is most similar ( $\sim 31 \%$ identity/49\% similar) to a 1189 aa predicted human protein (KIAA1355) that also contains five Ig domains, two Fn domains, and one predicted TM region (Fig. 2). This protein was identified in a large-scale human cDNA cloning project in which cDNAs were constructed from an RNA template that was extracted from specific human tissues (Nagase et al., 1999). The KIAA1355 transcript is expressed at high levels in fetal brain tissue (Nagase et al., 1999). Significant global amino acid homology also exists between Tutl and Neo from several phyla (26\% identity/39\% similarity to rat Neogenin) as well as to Robo from several phyla (25\% identity/40\% similarity to Dro- sophila Roundabout I; Fig. 2). However, the functional domain structures of the Neo and Robo subfamilies are significantly different from Tutl, with four Ig domains and six Fn domains (Vielmetter et al., 1994) and five Ig domains and three Fn domains (Kidd et al., 1998), respectively. We conclude that Tutl is related to the Neo and Robo families of receptors but is significantly divergent from either in both primary sequence and domain structure. In addition, Tutl is identical to the FasII protein in its domain structure (five Ig domains followed by two Fn domains), but the primary structure of these two proteins is highly divergent. The FasII protein is not retrieved in the top 100 most similar proteins in a sequence alignment search, suggesting that the similar domain structure arose through convergent evolution rather than divergence from a recent common ancestor. Thus, Turtle represents a novel Ig family within the Ig transmembrane receptor superfamily.

\section{The tutl gene has an analog adjacent on chromosome 2}

The closest relative of the tutl gene within the Drosophila genome is the Berkeley cDNA (BcDNA) GH11322 gene located adjacent to the tutl locus, with its primary transcription start site located $1.2 \mathrm{~kb}$ downstream of tutl (see Figs. $1 A, 4 A$ ). This arrangement suggests that the paired genes may have arisen from a tandem duplication event. The full-length cDNA sequence of BcDNA GH11322 was analyzed for structural similarity to tutl. BcDNA GH11322 produces a 719 aa protein product that contains four Ig domains, two Fn domains, and one TM region with similar domain structure and orientation to tutl Ig domains (1-3 and 5), Fn domains (1-2) and the TM region. The BcDNA GH11322 protein, however, is quite divergent from Tutl in primary struc- 


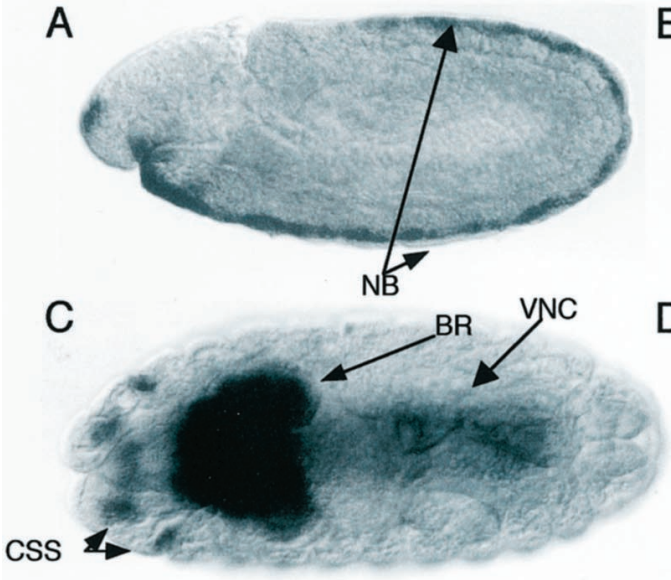

E
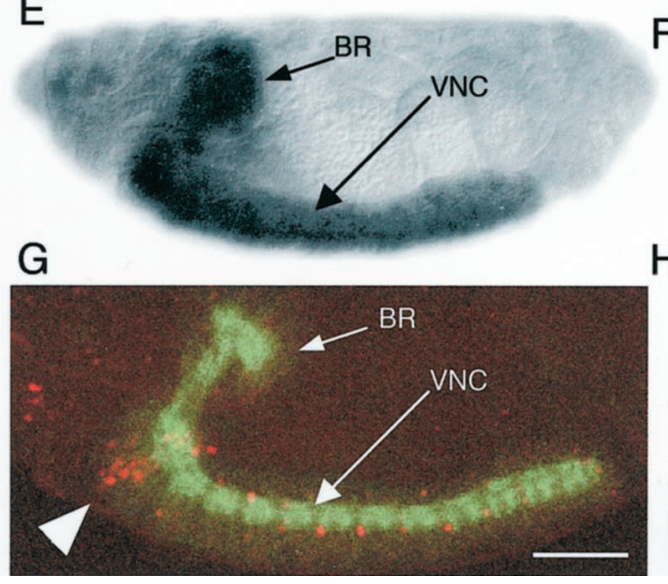

B
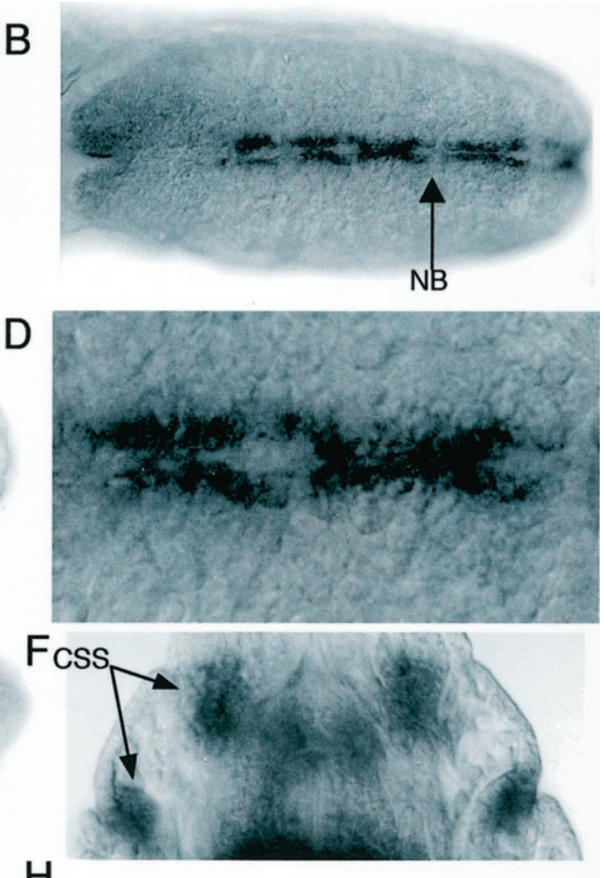

$\mathrm{H}$

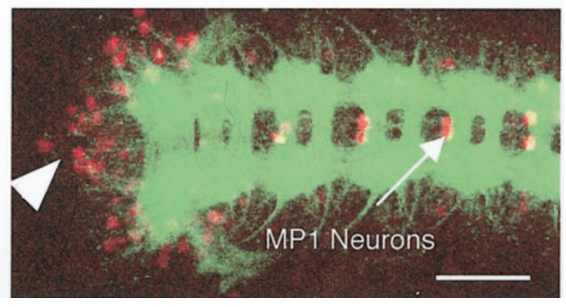

Figure 3. Expression of tutl during embryonic development. $A-F$, Developmental progression of staged Drosophila embryos stained to reveal tutl mRNA expression. Embryos are oriented with anterior to the left $(A-E, G, H)$ or top $(F)$; dorsal at top $(A$, $E, G)$, facing out $(B-D, F)$, or facing in $(H)$. $A$, Stage 9, onset of tutl expression in neuroblasts recently delaminated from neuroectoderm. $B$, Stage 9 embryo showing expression along developing CNS midline. $C$, Stage 16, tutl expression is limited to the CNS and the CSS. D, Enlargement of the neuroblast expression indicated by an arrow in $B$. E, Stage 16, strong tutl expression throughout the brain and ventral nerve cord. No detectable expression in nonneuronal tissues was observed. $F$, Enlargement of cephalic sensory structures shown with arrow in $C$. $G-H$, Expression of a tutlspecific reporter gene. $G$, Stage 16, 1(2)01085 $\beta$-gal expression (red) driven by the endogenous tutl enhancer. The CNS was stained with mAb BP102 for reference purposes (green). $H$, Tutl reporter expression is confined to the CNS with high-level expression in the MP1 interneurons and a cluster of cell bodies at the anterior portion of the ventral nerve cord (arrowhead). BR, Brain; $N B$, neuroblast(s); $V N C$, ventral nerve cord. Scale bars: $A, C, E, G, 90 \mu \mathrm{m} ; B, H, 60 \mu \mathrm{m}$; $D, 20 \mu \mathrm{m} ; F, 35 \mu \mathrm{m}$. ture (32\% identity/50 similarity; Fig. 2B). BLASTP scores comparing Tutl with the BcDNA GH11322 protein were slightly lower than scores comparing Tutl and the human brain protein KIAA1355 (Fig. 2B). The divergent nature of tutl and BcDNA GH11322 suggests that the spatial relationship could be coincidental. If a duplication event gave rise to the two genes, it likely occurred $>600$ million years ago, before the evolutionary divergence of Drosophila and Homo sapiens (Rodakis et al., 1984).

Despite the protracted divergence of tutl and BcDNA GH11322 genes, the similar domain structure and possible common evolutionary origin suggest that some functional redundancy may exist between their protein products. To investigate this possibility, we included the tutl 4 mutant allele in all experiments described below; tutl4 is a chromosomal deficiency that removes both tutl and BcDNA GH11322 genes (see Fig. 4A). As will be described in detail below, we found that tutl 4 mutants do not exhibit any phenotypes that are not present in mutations specific to the tutl gene. This analysis provides genetic evidence that the BcDNA GH11322 gene is not masking any functional roles or mutant phenotypes of tutl.

\section{turtle is expressed exclusively in the nervous system}

In situ hybridization studies using digoxygenin-labeled DNA probes complimentary to both exons $1-4$ and exon 6 were conducted to determine the temporal and spatial profile of tutl expression. The expression of tutl is restricted to the nervous system during all stages of development (Fig. 3). tutl mRNA expression begins at embryo stage 9 in midline neuroblasts that have recently delaminated from the neuroectoderm (Fig.
$3 A, B, D)$. From stage 10 to the end of embryogenesis, tutl mRNA is detected in a pan-neural pattern throughout the CNS (Fig. $3 C, E)$. Beginning at stage 13 , four clusters of cells representing cephalic sensory structures (CSS) at the extreme anterior portion of the animal begin to express the tutl transcript and continue to do so throughout embryogenesis (Fig. 3C,F). tutl expression in this very small, defined subset of the sensory PNS was the only detectable expression outside of the CNS. There was no detectable tutl expression before stage 9, and no tutl expression was detected in any non-neural tissues or cell types during embryogenesis. We conclude, therefore, that tutl is expressed throughout the CNS from segregation of neuronal precursors through the end of development and serves in functions specific to the CNS and a small subset of sensory neurons in the head.

In addition to tutl neural expression during embryogenesis, we found that the tutl transcript continues to be expressed through larval, pupal, and adult stages. cDNA clones specific to tutl were present in mRNA isolated from embryos (clone LD28224), larvae and early pupae (LP03459), and adult head (clones GH15753, GH16705, GH08133, and HL01565), confirming that the gene is expressed in all stages of development and maturity. In addition, a lacZ reporter gene that is present in the tutl 1(2)01085 P-element insertion (see below) is expressed from embryogenesis (beginning in stage 12) to adult stages (Fig. $3 G, H$ ). The spatial expression of the tutl reporter is identical to tutl mRNA expression, both confined to the CNS and a small subset of head PNS from embryonic through larval stages. However, the reporter expression more sensitively detects differences in tutl expression levels 


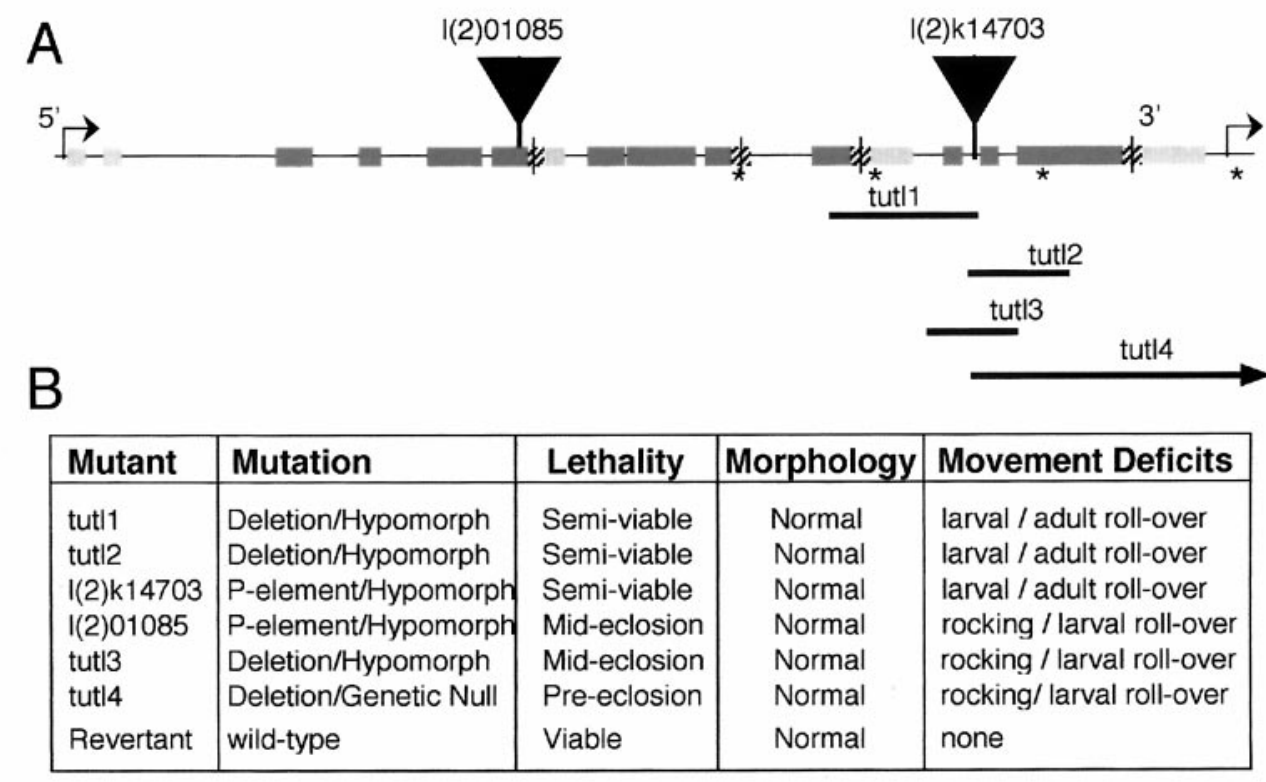

Figure 4. Characterization of mutations in the tutl gene. A, Genomic structure of the tutl gene and location of tutl mutations (see Fig. 1 for symbol legend). Asterisks indicate locations of primer sets (\#6, \#7, \#8, and \#9) used for PCR mapping of deletion breakpoints in tutl1, tutl2, tutl3, and tutl4 mutations. Note that the tutl4 deletion extends $3^{\prime}$ to remove the transcriptional region of the BcDNA GH11322 gene. Vertical arrowheads indicate insertion sites of P-elements that generated l(2)01085 and 1(2)k14703 mutations. B, The six tutl mutations generated in this study and the genetic rescue (revertant) with details about mutation type, lethal stage of homozygous mutants, general morphology of larvae (and adults for semiviable mutants), and movement and behavioral phenotypes. (See Results for details.)

within the CNS (Fig. 3G,H). Particularly high levels of tutl expression are found in the MP1 interneurons, which cross the midline and project posteriorly, as well as in a group of unidentified neurons at the anterior border of the ventral nerve cord late in embryogenesis (Goodman et al., 1984) (Fig. 3G,H). This CNS-specific expression continues into adult stages, which suggests a functional role for tutl that begins in embryogenesis but continues to play an essential role in maintaining a normal mature nervous system (Mollereau et al., 2000).

\section{turtle function is essential in Drosophila}

We identified two recessive lethal P-lacZ insertions (1(2)01085 and $1(2) \mathrm{k} 14703)$ at 24E1-3. These two inserts fail to complement one another in lethal complementation tests, and both are inserted in the tutl coding region (Fig. 4A). The 1(2)01085 P-element is inserted within the boundaries of exon 4 (Fig. 4 ) and disrupts translation of the tutl transcript downstream of exon 4 , possibly silencing the entire gene. The $1(2) \mathrm{k} 14703$ insert is located between exon 8 and exon 11 and produces a mutation similar to the 1(2)01085 as defined by similarity in the types and severity of the mutant phenotypes (see below).

The 1(2)k14703 P-element was mobilized to produce both precise and imprecise excision events (see Materials and Methods). Precise excision events (revertants) were isolated and were shown to completely rescue the lethal phenotype associated with this mutation (Fig. 4B) as well as all other phenotypes characterized here. Precise excision of this P-element accomplishes genetic rescue of the tutl gene and confirms that insertional mutations in the tutl gene are responsible for the lethal phenotypes described below. In addition, four imprecise excision events were isolated (tutl1-4) that contain Dfs of varying lengths; these Dfs produce phenotypes of increasing severity in the tutl gene. The extent of each deficiency (Fig. 4A) and the relative severity of associated phenotypes (Fig. 4B) are shown.

In total, six recessive lethal mutations of the tutl gene were isolated and characterized in this study (Fig. 4). Most animals homozygous for the tutl1, tutl2, or 1(2)k14703 mutations are able to survive to adulthood if competition with heterozygous siblings is eliminated by isolated rearing of isogenic mutants. However, pressure from siblings carrying one wild-type copy of tutl results in the death of the homozygous animals (85-100\% lethality) before adulthood. The 1(2)01085, tutl3, and tutl 4 mutants, even in the absence of competition with siblings carrying wild-type tutl, die $100 \%$ of the time during late pupation shortly before or during hatching from their pupal cases. These studies show that tutl is absolutely required for nervous system development and adult viability.

\section{turtle is required for specific types of coordinated movement}

Mutations in the turtle gene produce obvious, profound defects in several behaviors. We assayed coordinated movement using a variety of paradigms from stage L1 through adulthood (Fig. 5). We initially observed that tutl mutants react abnormally to tactile stimulation. When wild-type larvae are touched at their anterior end, they respond by executing a characteristic tight reverse-andturn escape response. This escape behavior is characterized by one to three reverse peristalsis movements followed by a lateral turning behavior. Escape behaviors such as this are robust and reliable to the extent that investigators have successfully conducted genetic screens based on variations in this mechanosensory response (Kernan et al., 1994). 1(2)01085, tutl3, and tutl4 mutant larvae react to this stimulus test abnormally. Mutant animals always respond to tactile stimulation by contracting at 


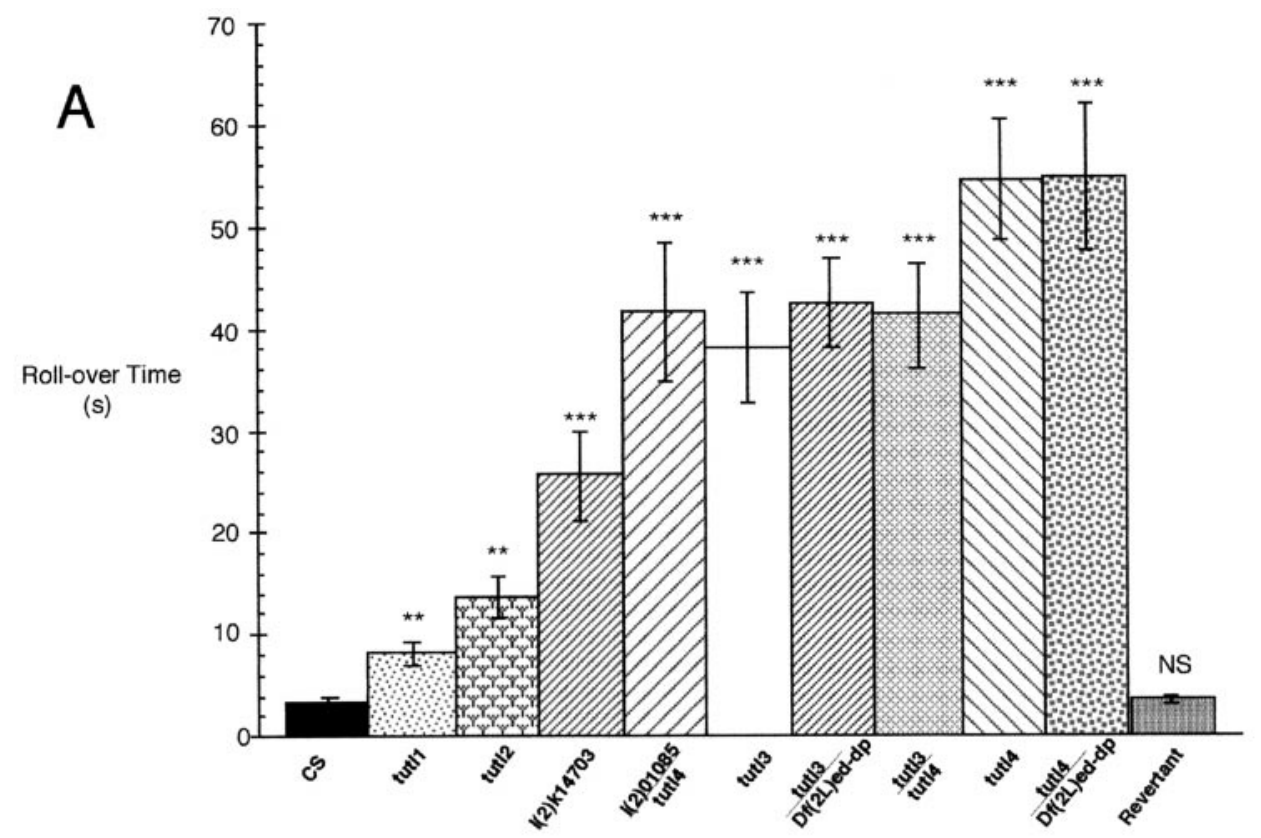

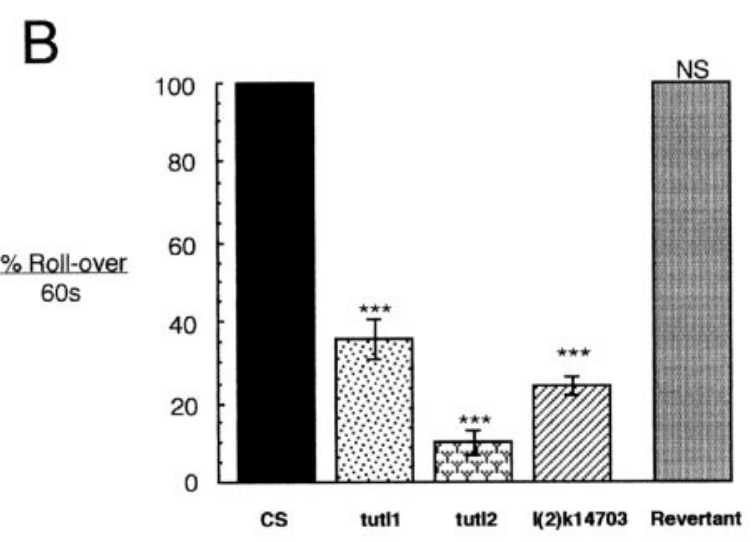

both ends and rocking back and forth in place, which results in little or no net displacement of the animal. After a few seconds of this behavior, the larvae will cease this abnormal movement and return to a normal forward-crawling motion, failing to execute any escape behavior. This profound behavioral defect is absent in revertant larvae and therefore is a result of a mutation in the tutl gene. This response defect demonstrates an inability of tutl mutants to coordinate the specific sequence of motor outputs required for coordinated, bilateral behavior.

We followed this qualitative observation with quantitative studies designed to reveal the severity of behavioral defects. Wildtype larvae placed in an inverted position will always respond by executing a twist-and-roll behavior that will enable the animal to rapidly right itself. This response clearly requires coordinated bilateral motor control, allowing the animal to contract muscles on one side while relaxing contralateral muscles. We analyzed this larval behavior in homozygous tutl mutants to determine whether the larvae exhibited defects in this type of complex coordinated movement. We found that tutl mutants exhibit a severely compromised ability to execute the coordinated motor output necessary to roll over from an inverted position (Fig. $5 A$ ). Wild-type controls always completed the maneuver in $<5$ $\mathrm{sec}$, and all tutl mutants were significantly delayed ( $>10 \mathrm{sec})$.
Figure 5. Behavioral assays of tutl mutants. $A$, Time required for $\mathrm{L} 3$ larvae to roll from an inverted to an upright position (in seconds) for CS (wild-type control), tutl Df mutants (tutl 1 , tutl 2 , tutl 3 , tutl 4 ), and P-element tutl mutants (1(2)k14703, 1(2)01085). B, Percentage of adult flies that can roll from an inverted to an upright position in a $60 \mathrm{sec}$ time period. $C$, The number of peristalsis contraction waves during normal locomotion executed by L3 larvae in a $60 \mathrm{sec}$ time period. $n=15$ for all lines in $A$ and $C ; n=5$ groups of 10 adults in $B$. Error bars indicate the SEM. Asterisks indicate the degree of significance relative to wild type in degrees of magnitude of the $p$ value.

Each of the six tutl mutants exhibits this phenotype to a different degree: tutl 3 increases roll-over time by $\sim 10$-fold ( 31 sec), whereas tutl4 as well as tutl4/Df(2L)ed-dp flies delay this behavior by nearly 20 -fold $(55 \mathrm{sec}$ ) (Fig. $5 A$ ). The identical phenotype of tutl4/tutl4 and tutl4/Df demonstrates that tutl4 is a null mutation by genetic criteria. The other tutl alleles, with weaker phenotypes, represent hypomorphic partial loss of function mutations. Importantly, precise excision of the 1(2)k14703 P-element insertion completely rescues this phenotype to wild-type levels (Fig. $5 A$ ).

To determine whether defects in these complex behaviors are secondary to a more general problem, such as a severe lack of energy or severe sluggishness, lethal mutants were analyzed for basal movement and locomotion behavior. We found that tutl mutant larvae executed peristalsis waves during crawling locomotion at only slightly reduced rates relative to wild type (Fig. 5C). Indeed, the tutl genetic null mutant (tutl4), which rolls $>20$-fold slower than wild type, is only slightly hindered $(<15 \%)$ in its rate of executing peristalsis contraction waves during normal locomotion (Fig. 5, compare $A$ with $C$ ). Although normal movement is comparable between mutant and control animals, more complex migratory behavior is again markedly abnormal. Whereas wildtype larvae move in a continuous, linear direction when exploring 

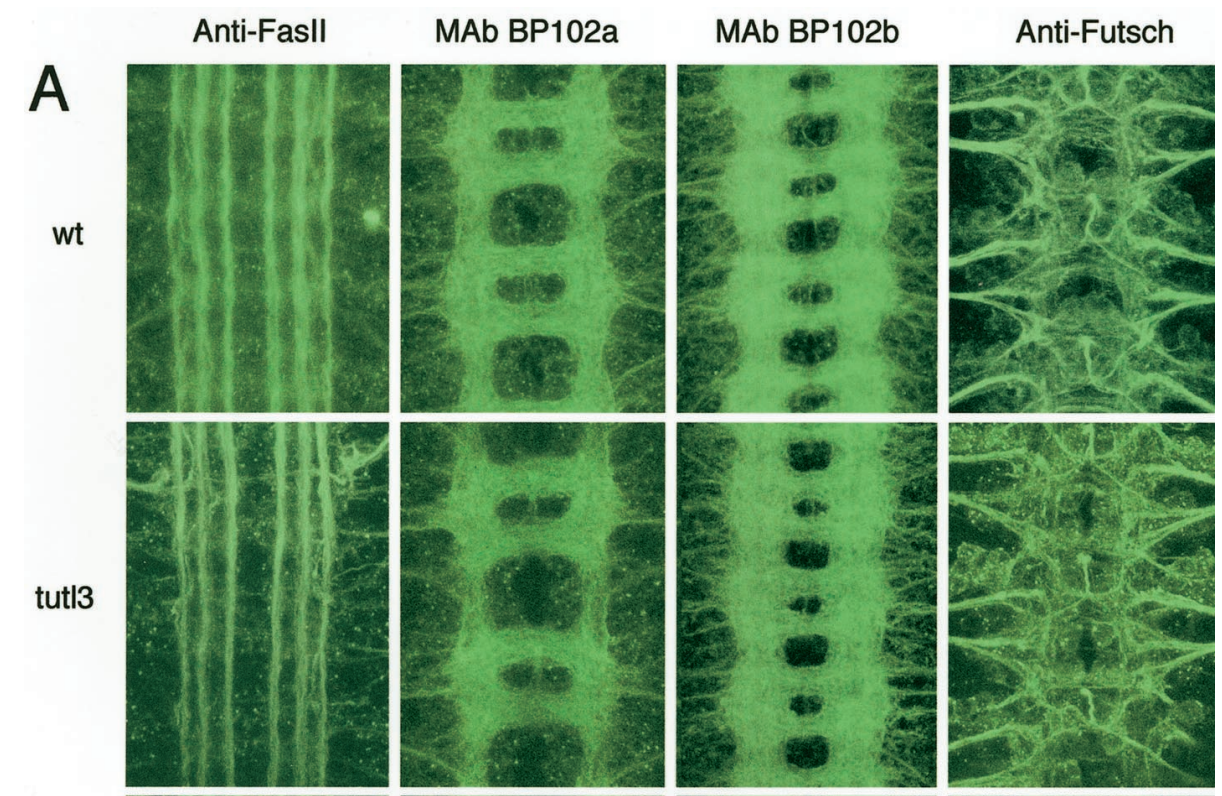

Figure 6. No morphological defects detected in the embryonic nervous system of tutl mutants. $A$, Confocal images of the ventral nerve cord in the CNS of CS wild-type (wt), strong hypomorph tutl3, and null tutl4 mutants. Anti-FasII, The three longitudinal axon tracts on each side of the midline of stage 16 embryos visualized with $\mathrm{mAb}$ 1D4. No abnormalities were detected in these structures. $m A b$ BP102a, Commissural and longitudinal axons in the CNS of stage 14 embryos visualized with mAb BP102. No disruption of axon scaffold segments was found. $m A b B P 102 b$, Commissural and longitudinal axons in the CNS of stage 16 embryos visualized with mAb BP102. Development of the CNS appears to progress normally in tutl mutants throughout embryogenesis. Anti-Futsch, Motor neuron axon tracts including VUM axons, segmental nerves, and intersegmental nerves visualized with $\mathrm{mAb} 22 \mathrm{C} 10$. No abnormalities were detected in these pathways. $B$, Confocal images of the CSS in which tutl mRNA is expressed (Fig. 3C,F). The CSS of wild-type (wt) and tutl 4 stage 16 embryos are visualized with mAb 22C10 (anti-Futsch). The morphology of these structures is normal.

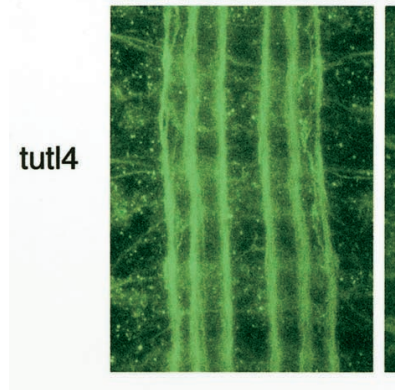

wt
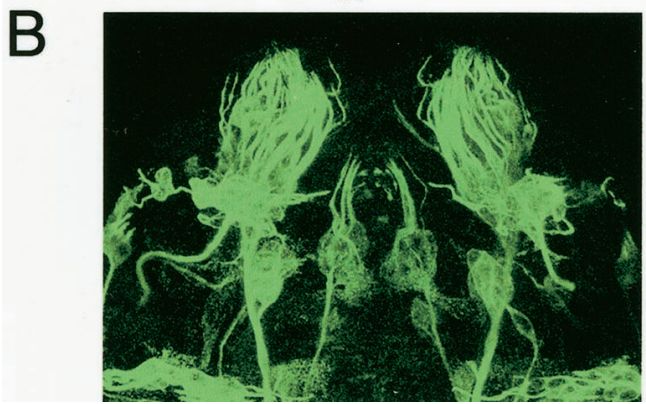
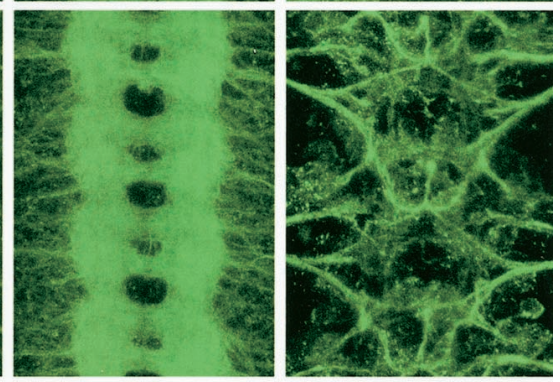

tutl4

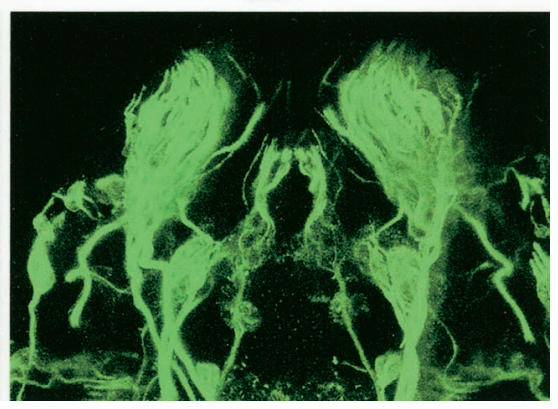

a clean agar plate, tutl mutants stop and turn constantly and so fail to move significantly in any given direction. These data again demonstrate that tutl mutant larvae are able to execute basal, locomotory movement similar to wild type but are severely impaired in their ability to execute complex types of coordinated behavior.

Isogenic homozygous tutl1, tutl2, and 1(2)k14703 mutants, the only mutants that can survive to adult stages, are also drastically impaired in their ability to execute specific types of adult coordinated movement. Most strikingly, these adult animals are completely unable to perform flight of any kind, although the animals walk normally and energetically on a level surface. In addition, the jump response of these adults is intact and robust, but uncoordinated attempts at flight result in the flies flipping themselves onto their backs, where continued efforts to fly are exhibited by adults flapping their wings at high speeds. These efforts, however, only result in the flies spinning frantically in circles on the table top. This behavior demonstrates well developed and well innervated flight muscles in tutl mutants but the inability to coordinate the motor control required for this complex behavior. In addition, tutl mutant adults that become inverted are rarely able to right themselves despite what appears to be an intense effort to roll over. This behavior was quantified by manually inverting individuals and assaying righting behavior for $60 \mathrm{sec}$. As you might expect, wild-type flies always roll over and escape immediately, almost always in $<1 \mathrm{sec}$. In sharp contrast, only $12-36 \%$ of tutl 1 , tutl2, and l(2)k14703 adults are able to roll over after a full $60 \mathrm{sec}$, whereas revertant adults are indistinguishable from wild type, usually escaping in $<1 \mathrm{sec}$ (Fig. $5 B$ ). Thus, as in the larvae, righting behavior is severely compromised in tutl adult mutants. The inability to fly and roll over in adulthood appears to result from a compromised ability to execute specific kinds of bilateral, coordinated movement. This striking phenotype gave rise to the mutant name, "turtle". 


\section{Canton-S}

A
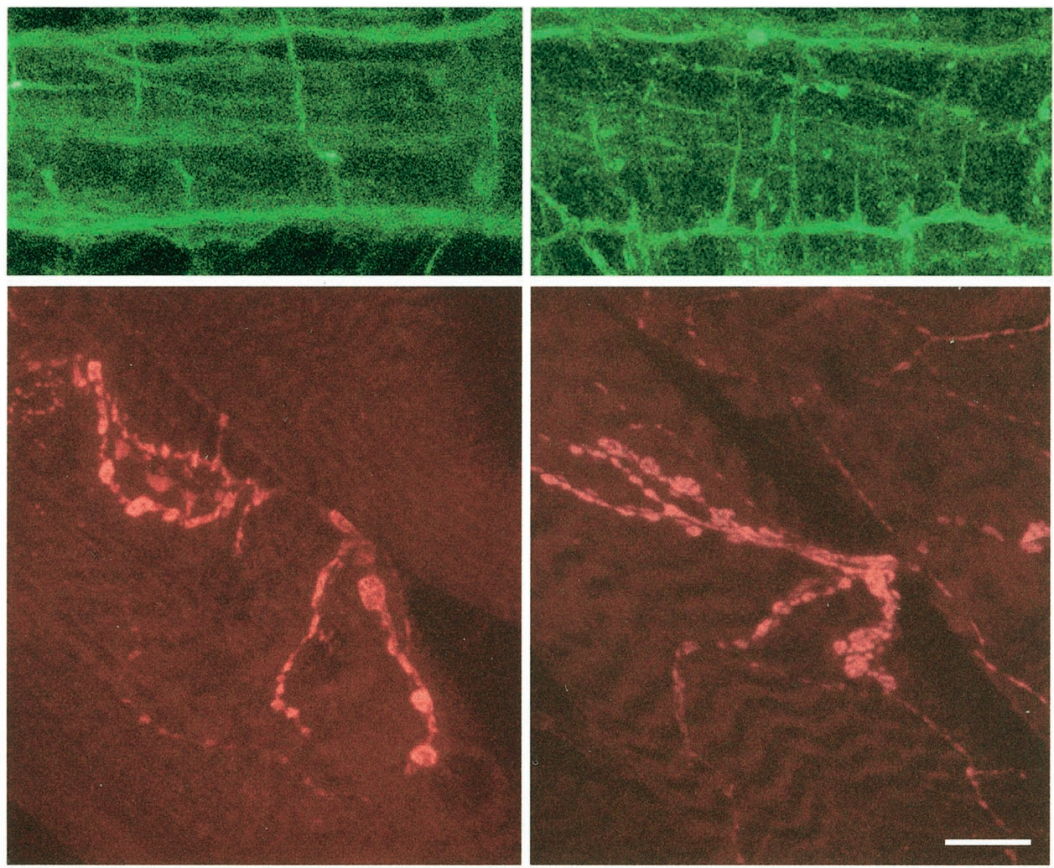

Canton-S

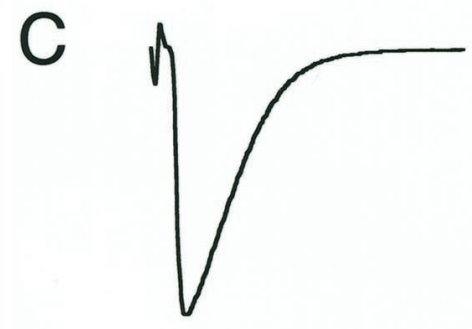

tut14

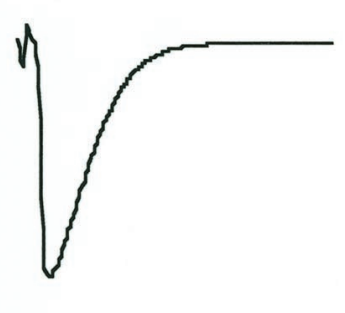

D

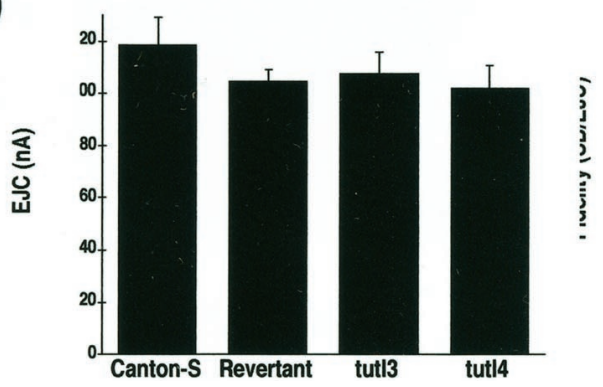

Revertant

tutl4
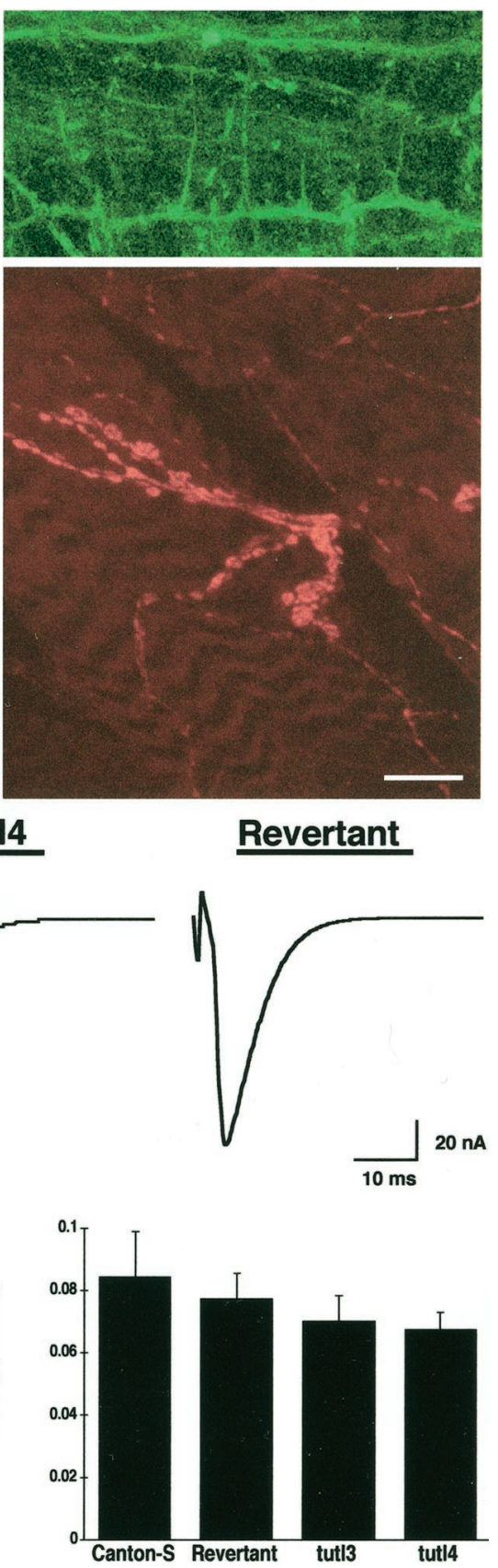

Figure 7. No morphological defects were detected in the larval CNS. The structure and function of the NMJ is also normal. $A$, The CNS in L3 wild-type and tutl4 larvae visualized with $\mathrm{mAb}$ 1D4 (anti-FasII). The three longitudinal axon tracts of the CNS on each side of the midline are shown. No discontinuity or abnormality was found in these structures in null tutl 4 larvae. $B$, The NMJ of muscle 12 in L3 wild-type and tutl4 larvae visualized with antisynaptotagmin. Presynaptic morphology appears to be normal. $C$, EJC amplitude and response kinetics are unaffected by tutl. Each trace represents an average of individual responses from control (7 traces), mutant (8 traces), and revertant (10 traces). D, Basal EJC fidelity and amplitude are unaffected by mutations in tutl. Left, EJC amplitude at $0.5 \mathrm{~Hz}, 0.4 \mathrm{~mm}$ bath $\left[\mathrm{Ca}^{2+}\right]$. Right, fidelity of response measures variability of response within the individual muscle and is unchanged by tutl mutation. For each line, $n=9$. Error bars indicate the SEM. Categories were compared by nonparametric ANOVA and differences were not significant (left, $p>0.6821$; right, $p>0.8412)$. Scale bars: $A, 35 \mu \mathrm{m} ; B, 30 \mu \mathrm{m}$.

\section{Turtle does not detectably mediate neuronal morphogenesis}

Because of the similarity between the primary structure of Tutl and the Neo/Fra and Robo/Dutt1 protein families, we suspected that Tutl might function in a similar mannor to mediate growth cone guidance and neuronal pathfinding. Specifically, Tutl is structurally similar to chemotrophic receptors known to be present on the surface of growth cones that navigate midline crossing in the CNS (for review, see Tear et al., 1993; Hummel et al., 1999). Such a midline guidance function for Tutl would be consistent with the observed profound defects in bilateral motor control.

We used multiple probes for immunocytochemistry and confocal laser-scanning microscopy to image the developing CNS in mutant embryos and larvae for pathfinding or neuronal wiring defects (Fig. 6). We first analyzed the axon scaffold in the embryonic CNS of severe hypomorphic tutl3 and tutl4 genetic null mutants stained with mAb BP102 (Seeger et al., 1993), which recognizes an unknown epitope present at the axon scaffold of the CNS (Fig. 6A). We detected no abnormalities in the morphology of the longitudinal tracts or the anterior and posterior commissures in the axon scaffold (Fig. 7A). Analyses at different stages (stage 9-17) of neuronal development throughout embryogenesis similarly failed to detect any transient defects in axonal patterning. Overall embryonic CNS structure stained with mAb BP102 was also visualized to analyze brain morphology, axon scaffold segment size, and axon scaffold segment shape, but no variation from wild type was detected. 
We subsequently analyzed CNS structure using mAb 1D4, which recognizes the FasII protein (Vactor et al., 1993), and mAb 22C10, which recognizes the Futsch protein (Fujita et al., 1982; Hummel et al., 2000), to visualize subsets of axon tracts in the CNS that were not distinguishable using mAb BP102. We found no abnormalities in the three FasII-positive longitudinal fascicles on either side of the midline or in the motor axon pathways that exit the CNS to innervate the musculature (Fig. 6A). Using mAb $22 \mathrm{C} 10$, we visualized the ventral unpaired median (VUM) axons, which originate in the midline, project through the posterior commissure to the anterior commissure, and then bif urcate and turn to leave the CNS. We also visualized the morphology of the segmental and intersegmental nerves from which axon bundles exit the CNS, providing pathways for motor axons including VUM axons (Goodman et al., 1984). No disruption or abnormality of the axon tracts, segmental nerves, or intersegmental nerves in the CNS was detected in tutl mutants (Fig. 6A). From these analyses, we conclude that the Tutl protein does not play a major role in axon pathfinding, either across the midline of the CNS or between neuromeres in the $\mathrm{CNS}$, or in regulating motor neuron projections from the CNS.

Because of the tutl expression detected in a limited subset of head PNS (Fig. 3C,F-H), we investigated the morphology of these tutl-positive cells that we call CSS using mAb 22C10 (Fig. $6 B)$. We found no morphological abnormalities in these structures in tutl3 or tutl 4 mutants, suggesting that the tutl protein does not play a detectable role in the morphological development of the CSS. The remainder of the PNS was also analyzed for morphogenic abnormalities, but none were detected; images that illustrate these analyses are not included because tutl is not expressed in these structures. Thus, at this high level of resolution, Turtle does not appear to play a role in the embryonic morphogenesis of either the CNS or PNS and, specifically, does not cause detectable structural defects in neurons known to express a high level of tutl. We cannot, of course, exclude the possibility the tutl plays a pathfinding role in a small subset of essential neurons not detectable with these techniques.

Mutations in turtle result in postembryonic lethality. Therefore, we investigated the possibility that morphological defects might arise only postembryonically in the CNS of tutl mutant larvae. We found that the gross morphology of the brain, ventral nerve cord, and motor nerves in tutl4 L3 larvae was normal. To investigate axon tracts at the L3 larval stage, we used mAb 1D4 to stain the FasII-positive longitudinal axon tracts of the ventral nerve cord in tutl4 larvae (Fig. 7A). We found that these structures similarly exhibit no abnormalities or defects. Therefore, we conclude that tutl does not play a detectable role in neuronal pathfinding or morphogenesis during postembryonic larval development. To be comprehensive, we extended our analyses to investigate the morphology of the neuromuscular junction (NMJ) by visualizing the neuromusculature of dissected L3 tutl4 larvae. We stained NMJs with anti-synaptotagmin antibody that recognizes a TM protein in synaptic vesicles (Littleton et al., 1993) and then analyzed type-I and type-II bouton morphology on muscle 12 (Fig. 7B). These analyses revealed no abnormalities in muscle innervation or presynaptic NMJ morphology in tutl mutants. We conclude that tutl does not play a detectable role in the elaboration of peripheral synaptic terminals used in movement regulation.

\section{turtle mutants have normal basal synaptic function}

Movement or behavioral defects can result from abnormal synaptic transmission. CNS synapses in Drosophila are, as yet, inaccessible to electrophysiology experiments, but the NMJ has been used extensively as a functional model for studying synaptic physiology (Littleton et al., 1999). Because of the severe behavioral phenotypes that are exhibited by tutl mutants, we suspected that a possible defect in synaptic transmission might contribute to the behavioral phenotypes exhibited by these mutants. We investigated this possibility by conducting electrophysiology assays to measure synaptic transmission at the larval NMJ.

Using TEVC configuration, we recorded evoked synaptic currents in control and tutl4 genetic null L3 larvae (Fig. 7C,D). The motor nerve was stimulated with a suction electrode, and synaptic excitatory junctional currents (EJCs) were recorded in the muscle. Qualitatively, we could detect no differences in synaptic transmission between mutants and controls (Fig. $7 C$ ). We found that the kinetics as well as the amplitude of EJCs at the NMJ are not significantly different from wild type (Fig. 7D). We conclude from these data that tutl is not essential for basal excitatory synaptic communication.

\section{turtle is expressed in localized regions of the adult brain}

turtle genetic null mutants die at the end of pupal development, suggesting that the essential function of the gene is not manifested until adult stages. Therefore, we examined the expression and function of tutl in the adult brain. Both tutl mRNA and a tutl $l a c Z$ reporter gene are expressed in similar patterns in the adult brain (Fig. $8 A-D$ ). High levels of tutl expression are observed only in highly localized subsets of neurons in the adult brain, in contrast to the more global expression observed in the embryonic CNS. One area of particularly high expression is in the region of the superior lateral protocerebrum (slpr), a brain region whose function has not yet been elucidated (Fig. 8A,C). In situ hybridization against tutl in adult frontal brain slices confirms this relatively confined expression pattern for tutl (Fig. 8D). Therefore, tutl expression is maintained in the adult brain, which suggests a functional role for tutl in maintaining a normal mature nervous system.

Because pronounced tutl expression is observed in specific brain regions (Fig. $8 A-D$ ) and profound behavioral defects continue to be exhibited in viable adult mutant animals (Fig. $5 B$ ), we investigated the morphological integrity of the adult CNS by staining sequential $7 \mu \mathrm{m}$ slices of the adult brain with $\mathrm{mAb}$ BP102. This antibody nicely reveals the architecture of the adult brain and specifically highlights regions with known tutl expression (Fig. $8 E-H$ ). First, we analyzed the gross morphology of all of the major adult brain regions (Fig. $8 C, E, G$ ). Figure $8, E$ and $G$, represents a sample of frontal brain histology slices used to analyze adult brain structure; however, sequential slices through the entire brain were used in our investigation. We detected no alterations in brain morphology in the viable tutl mutants. Second, we focused on structures that highly express tutl, including the superior lateral protocerebrum and regions associated with the mushroom bodies. However, we similarly observed no detectable alteration in neuronal architecture in these regions (Fig. $8 F, H)$. Together, these results show that turtle plays an essential role that is required for the manifestation of complex behaviors but that this defect is not associated with detectable alterations in neuronal morphology. We conclude that turtle is the founding 

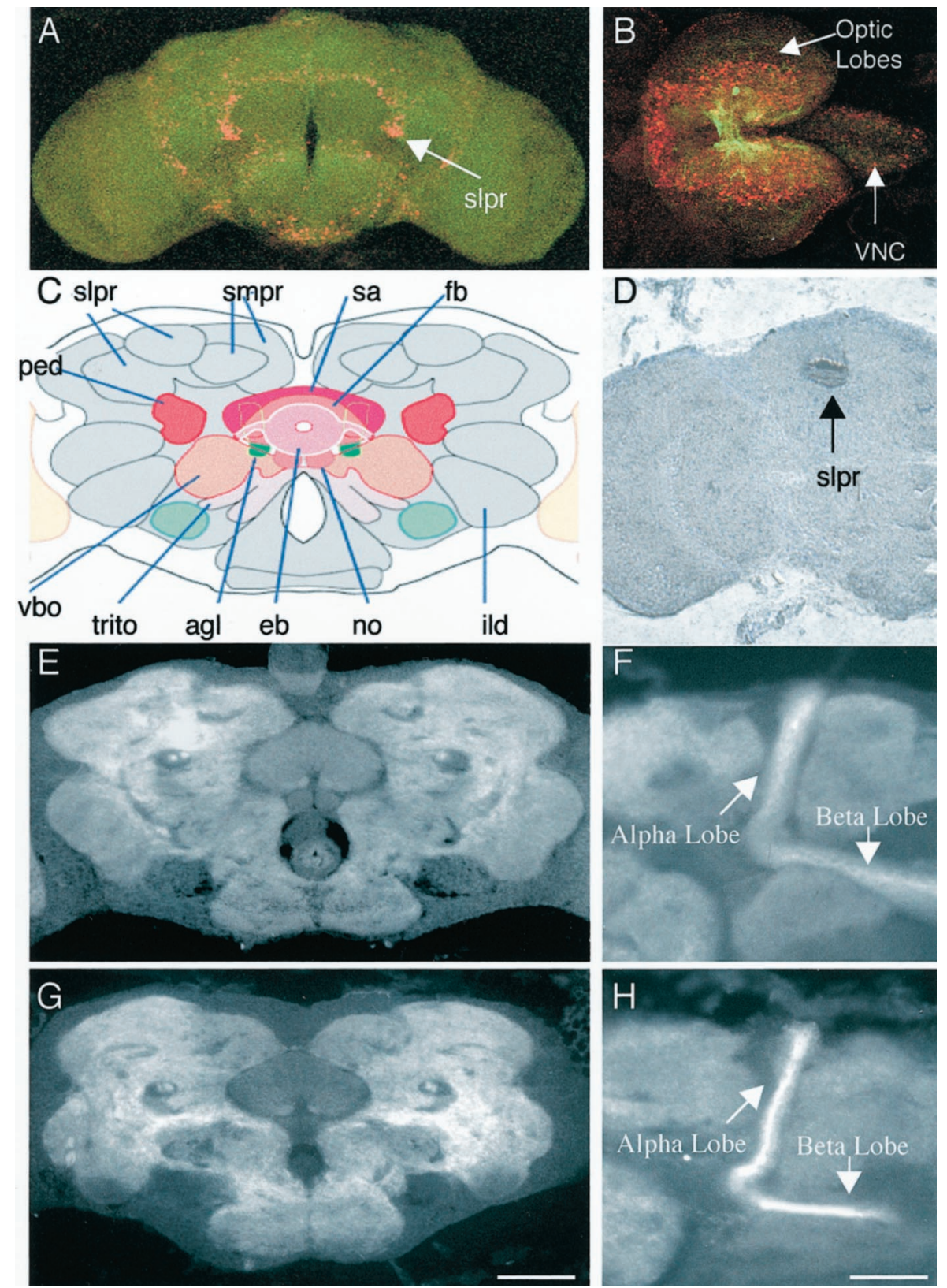

Figure 8. tutl is expressed in the larval and adult CNS, but no morphological defects were detected in these structures. $A, B$, tutl reporter gene expression in the adult brain and the larval brain represented by $\beta$-gal expression from the lac $Z$ gene on the 1(2)01085 P-element (red). The CNS was stained with mAb BP102 for reference purposes (green). Oriented dorsal out of the page and anterior up $(A)$ or to the left $(B)$. $C$, Frontal schematic of an adult Drosophila brain from the Flybrain website (www.flybrain.org). ped, Pedunculus; $f b$, fan-shaped body; vbo, ventral body; smpr, superior medial protocerebrum; trito, tritocerebrum; sa, superior arch, eb, ellipsoid body; no, nodulus; ild, inferior lateral deutocerebrum; igt, antennoglomerular tract; vbo, ventral body. $D$, DNA in situ hybridization of a frontal adult brain histology slice demonstrating confined tutl expression in the adult brain to the region of the superior lateral protocerebrum. Note that this expression is consistent with that seen in $A$. E, Wild-type adult brain frontal slice stained with mAb BP102 to visualize structures labeled in $C$. F, Wild-type adult brain frontal slice stained with mAb BP102 to visualize the $\alpha$ and $\beta$ lobes of the mushroom bodies. $G, t u t l 2$ adult brain frontal slice stained with mAb BP102 to visualize structures labeled in $C$. No morphological abnormalities were found. $H$, tutl 2 adult brain frontal histology slice stained with mAb BP102 to visualize the $\alpha$ and $\beta$ lobes of the mushroom bodies. No morphological abnormalities were found in any of the lobes of the mushroom bodies. Scale bars: $A$, $100 \mu \mathrm{m} ; B, 90 \mu \mathrm{m} ; C-E, G, 60 \mu \mathrm{m} ; F, H, 30 \mu \mathrm{m}$. member of a neural-specific Ig family that plays a novel role in neuronal mechanisms.

\section{DISCUSSION}

We have identified and characterized a novel Drosophila gene, turtle, that is a member of the IgSF. The Turtle protein is most highly related to IgSF members Neogenin and Roundabout based on sequence homology and most related to IgSF member Fasciclin II based on Ig and fibronectin domain structure. The turtle gene produces at least four transcripts, including two transmembrane and two secreted isoforms. This molecular analysis suggested that Turtle may act as a cell-surface receptor, similar to other family members, but might have additional functions mediated through secretion.

We have shown that the turtle transcript is expressed exclusively, and at high levels, in the CNS and in a small subset of PNS sensory structures in the head. The expression of turtle begins in early embryonic neurogenesis, soon after neuroblasts delaminate from the neuroectoderm, and extends throughout the lifespan of the animal. The gene is widely expressed throughout the CNS but shows elevated levels of expression in a small subset of neurons in the embryonic nervous system and small regions of neurons in the larval and adult brain.

To investigate turtle function in the nervous system, we created an allelic series of mutants with P-element insertion and imprecise excision. We discovered that turtle is essential for viability, with genetic null mutants dying at the end of development before adult eclosion. The series of six turtle mutants all profoundly influence a number of complex behaviors requiring bilateral motor control. Mutants show defective exploratory behavior, escape response to tactile stimulation, and righting behavior, as well as an inability to coordinate flight. All behavioral defects were completely rescued in a P-element revertant, confirming the specificity of these turtle phenotypes. These behavioral defects do not occur because of a general problem, but rather appear specific 
for a number of complex behaviors requiring fine bilateral motor control.

Given the known function of related IgSF members, we focused our investigation based on the hypothesis that Turtle would have a function in axonal pathfinding during neuronal development. However, we found no axonal pathfinding or other neuronal morphogenic defects in any of the six turtle mutants in the embryonic, larval, or adult nervous systems. In these analyses, we used a number of immunological markers that reveal defects down to the single axonal level and have been used extensively in similar analyses to characterize pathfinding defects in related IgSF members. Nevertheless, it is possible that we missed a pathfinding function manifest in a small number of essential neurons or a small subset of neurons not revealed with any of our immunocytochemical markers. To be as comprehensive as possible, we also assayed synaptic structure and transmission properties in turtle genetic null mutants. However, turtle plays no detectable functional role in establishing synaptic morphology or mediating basal synaptic transmission, at least at the neuromuscular synapse, the only synapse currently available for analyses.

The lack of morphological defects in turtle mutants was surprising given the known function of its IgSF relatives, the Neogenin/Frazzled and the Roundabout/Dutt1 families. Both of these protein families have been found to function as cell-surface receptors that play critical roles in chemotrophic axon guidance mechanisms in the CNS (for review, see Tear et al., 1993; Kolodziej et al., 1996; Kidd et al., 1998; Hummel et al., 1999). In the absence of Fra or Robo in flies, striking axon pathfinding defects are apparent, especially in the axon scaffold of the ventral nerve cord and axonal midline crossing in the CNS (Kolodziej et al., 1996; Kidd et al., 1998; Sundaresan et al., 1998; Cooper et al., 1999). We conclude that Turtle, although a relative of known pathfinding receptors, plays a distinctive neuronal role. Although its mechanism of action remains unknown, Turtle is essential for viability and plays an essential role in mediating complex coordinated behavior that is independent of any detectable morphological wiring function in the CNS.

\section{turtle defines a novel family of the Ig superfamily}

The closest known relative of the turtle gene is the human gene KIAA1355 on chromosome 1, which was only recently identified based on mRNA isolated from fetal brain tissue (Nagase et al., 1999). This similarity is greater than that of turtle with its nearest relative in the Drosophila genome, an adjacent gene (BcDNA GH11322) predicted to have arisen from a duplication event before the divergence of Drosophila and human lineages. The fact that the closest known turtle relative exists in the evolutionarily distant human suggests that turtle and KIAA1355 define a completely novel subfamily of the IgSF that has not been studied previously. Although the turtle/KIAA1355 subfamily is identical to the Fasciclin II proteins in its domain structure, primary amino acid structure is very divergent. Thus, Turtle family members are likely to have functions distinct from the known Fasciclin II, Neogenin/Frazzled, and Roundabout/Dutt1 families.

\section{Does turtle have a novel function?}

Extensive investigation of an allelic series of turtle mutants involving assays of neuronal morphology, axon fascicle tracts, and domain structure in the embryonic through the adult nervous system has failed to reveal a detectable role in axon guidance. Therefore, based on the data presented here, we conclude that Turtle does not function by a developmental mechanism similar to its characterized relatives, but rather has a novel function that has not yet been resolved. We present evidence that turtle is required for specific kinds of complex behavior starting in the immediate postembryonic L1 stage and extending through adulthood. We found that certain types of complex, coordinated movements are severely hindered, whereas basal locomotion and behavior are only slightly altered or are completely unaffected. The specificity of these defects suggests that abnormalities exhibited in turtle mutants are not in the general machinery that mediates basic motor control; rather, the molecular defect most likely lies within a mechanism that enables Drosophila to execute complex coordinated behaviors.

Although we have yet to uncover the mechanism by which Turtle mediates complex behaviors, we feel that two possibilities are most likely. First, Turtle may play a role in axon pathfinding of a very small subset of neurons. The limited number, location, or uncharacterized function of this subset of neurons may have rendered them undetectable to us using current experimental techniques. Second, Turtle may function in a novel neuronal mechanism unrelated to other IgSF members. For example, given the known function of the structurally related Fasciclin II in mediating synaptic mechanisms, it is possible that Turtle function is essential to synaptic transmission in some neurons of the CNS. Investigation of this possibility is currently beyond the scope of available techniques. Additional analyses of turtle mutants in the fly as well as turtle homologs in other model organisms are required to reveal the mechanism by which this novel family mediates viability and behavioral regulation.

One exciting possibility is that turtle may be linked to an inherited human disorder that impairs coordinated behavior (Harding and Thomas, 1980). Several such inherited disorders have been defined only recently, and their mechanisms and genetic loci are not yet known (Koskinen et al., 1994; Fukuhara et al., 1995; Eckhardt et al., 1998). Model genetic systems in which we can study these human neurological disorders are invaluable and will likely contribute to our understanding of their pathologies and to the subsequent development of clinical therapeutics.

\section{REFERENCES}

Adams MD, Celniker SE, Holt RA, Evans CA, Gocayne JD, Amanatides PG, Scherer SE, Li PW, Hoskins RA, Galle RF, George RA, Lewis SE, Richards S, Ashburner M, Henderson SN, Sutton GG, Wortman JR, Yandell MD, Zhang Q, Chen LX, Brandon RC, Rogers YH, Blazej RG, Champe M, Pfeiffer BD, Wan KH, Doyle C, Baxter EG, Helt G, Nelson CR, Gabor GL, Abril JF, Agbayani A, An HJ, Andrews-Pfannkoch C, Baldwin D, Ballew RM, Basu A, Baxendale J, Bayraktaroglu L, Beasley EM, Beeson KY, Benos PV, Berman BP, Bhandari D, Bolshakov S, Borkova D, Botchan MR, Bouck J (2000) The genome sequence of Drosophila melanogaster. Science 287:2185-2195.

Ashburner M (1989) Mass histology of adult heads. In: Drosophila, a laboratory manual (Ashburner M, ed), pp 254-259. New York: Cold Spring Harbor Laboratory.

Barthels D, Santoni MJ, Wille W, Ruppert C, Chaix JC, Hirsch MR, Fontecilla-Camps JC, Goridis C (1987) Isolation and nucleotide sequence of mouse NCAM cDNA that codes for a $M_{\mathrm{r}} 79,000$ polypeptide without a membrane-spanning region. EMBO J 6:907-914.

Cooper HM, Gad JM, Keeling SL (1999) The Deleted in Colorectal Cancer netrin guidance system: a molecular strategy for neuronal navigation. Clin Exp Pharmacol Physiol 26:749-751.

Dickson B (1998) Axon guidance: a Roundabout way of avoiding the midline. Nature 391:442-443.

Eckhardt SM, Hicks EM, Herron B, Morrison PJ, Aicardi J (1998) New form of autosomal-recessive axonal hereditary sensory motor neuropathy. Pediatr Neurol 1998 19:234-235.

Fujita SC, Zipursky SL, Benzer S, Ferrus A, Shotwell SL (1982) Monoclonal antibodies against the Drosophila nervous system. Proc Natl Acad Sci USA 79:7929-7933.

Fukuhara N, Nakajima T, Sakajiri K, Matsubara N, Fujita M (1995) 
Hereditary motor and sensory neuropathy associated with cerebellar atrophy (HMSNCA): a new disease. J Neurol Sci 133:140-151.

Goodman CS (1996) Mechanisms and molecules that control growth cone guidance. Annu Rev Neurosci 19:341-377.

Goodman CS, Bastiani MJ, Doe CQ, du Lac S, Helfand SL, Kuwada JY, Thomas JB (1984) Cell recognition during neuronal development. Science 225:1271-1279.

Harding AE, Thomas PK (1980) Autosomal recessive forms of hereditary motor and sensory neuropathy. J Neurol Neurosurg Psychiatry 43:669-678.

Harrelson AL, Goodman CS (1988) Growth cone guidance in insects: fasciclin II is a member of the immunoglobulin superfamily. Science 242:700-708.

Huang Y, Jellies J, Johansen KM, Johansen J (1997) Differential glycosylation of tractin and LeechCAM, two novel Ig superfamily members, regulates neurite extension and fascicle formation. Cell Biol 138:143-157.

Hummel T, Schimmelpfeng K, Klambt C (1999) Commissure formation in the embryonic CNS of Drosophila. Dev Biol 209:381-398.

Hummel T, Krukkert K, Roos J, Davis G, Klambt C (2000) Drosophila Futsch/22C10 is a MAP1B-like protein required for dendritic and axonal development. Neuron 26:357-370.

Keino-Masu K, Masu M, Hinck L, Leonardo ED, Chan SS, Culotti JG, Tessier-Lavigne M (1996) Deleted in Colorectal Cancer (DCC) encodes a netrin receptor. Cell 87:175-185.

Kernan M, Cowan D, Zuker C (1994) Genetic dissection of mechanosensory transduction: mechanoreception-defective mutations of Drosophila. Neuron 12:1195-1206.

Kidd T, Brose K, Mitchell KJ, Fetter RD, Tessier-Lavigne M, Goodman CS, Tear G (1998) Roundabout controls axon crossing of the CNS midline and defines a novel subfamily of evolutionarily conserved guidance receptors. Cell 92:205-215.

Kohl A, Giese KP, Mohajeri MH, Montag D, Moos M, Schachner M (1992) Analysis of promoter activity and 5' genomic structure of the neural cell adhesion molecule L1. J Neurosci Res 32:167-177.

Kolodziej PA, Timpe LC, Mitchell KJ, Fried SR, Goodman CS, Jan LY, Jan YN (1996) frazzled encodes a Drosophila member of the DCC immunoglobulin subfamily and is required for CNS and motor axon guidance. Cell 87:197-204.

Koskinen T, Santavuori P, Sainio K, Lappi M, Kallio AK, Pihko H (1994) Infantile onset spinocerebellar ataxia with sensory neuropathy: a new inherited disease. J Neurol Sci 121:50-56.

Kunz S, Spirig M, Ginsburg C, Buchstaller A, Berger P, Lanz R, Rader C, Vogt L, Kunz B, Sonderegger P (1998) Neurite fasciculation mediated by complexes of axonin-1 and $\mathrm{Ng}$ cell adhesion molecule. J Cell Biol 143:1673-1690.

Littleton JT, Bellen HJ, Perin MS (1993) Expression of synaptotagmin in Drosophila reveals transport and localization of synaptic vesicles to the synapse. Development 118:1077-1088.

Littleton JT, Pallank L, Ganetzky B (1999) Mechanisms of neurotransmitter release. In: Neuromuscular junctions in Drosophila (Budnik V, ed), pp 139-162. San Diego: Academic.

Mollereau B, Wernet MF, Beaufils P, Killian D, Pichaud F, Kuhnlein R,
Desplan C (2000) A green fluorescent protein enhancer trap screen in Drosophila photoreceptor cells. Mech Dev 93:151-160.

Nagase T, Ishikawa K, Kikuno R, Hirosawa M, Nomura N, Ohara O (1999) Prediction of the coding sequences of unidentified human genes. XV. The complete sequences of 100 new cDNA clones from brain which code for large proteins in vitro. DNA Res 6:337-345.

Oster LL (1956) A new crossing-over suppressor in chromosome 2 effective in the presence of heterologous inversions. Drosophila Information Service 30:145.

Rodakis GC, Lecanidou R, Eickbush TH (1984) Diversity in a chorion multigene family created by tandem duplications and a putative geneconversion event. J Mol Evol 20:265-273.

Rohrbough J, Grotewiel MS, Davis RL, Broadie K (2000) Integrinmediated regulation of synaptic morphology, transmission, and plasticity. J Neurosci 20:6868-6878.

Schmucker D, Clemens J, Shu H, Worby C, Xiao J, Muda M, Dixon J, Zipursky S (2000) Drosophila Dscam is an axon guidance receptor exhibiting extraordinary molecular diversity. Cell 101:671-684.

Seeger M, Tear G, Ferres-Marco D, Goodman CS (1993) Mutations affecting growth cone guidance in Drosophila: genes necessary for guidance toward or away from the midline. Neuron 10:409-426.

Stoeckli ET, Kuhn TB, Duc CO, Ruegg MA, Sonderegger P (1991) The axonally secreted protein axonin-1 is a potent substratum for neurite growth. J Cell Biol 112:449-455.

Sundaresan V, Roberts I, Bateman A, Bankier A, Sheppard M, Hobbs C, Xiong J, Minna J, Latif F, Lerman M, Rabbitts P (1998) The DUTT1 gene, a novel NCAM family member, is expressed in developing murine neural tissues and has an unusually broad pattern of expression. Mol Cell Neurosci 11:29-35.

Tautz D, Pfeifle C (1989) A non-radioactive in situ hybridization method for the localization of specific RNAs in Drosophila embryos reveals translational control of the segmentation gene hunchback. Chromosoma 98:81-85.

Tear G, Seeger M, Goodman CS (1993) To cross or not to cross: a genetic analysis of guidance at the midline. Perspect Dev Neurobiol 1:183-194.

Teichmann SA, Chothia C (2000) Immunoglobulin superfamily proteins in Caenorhabditis elegans. J Mol Biol 296:1367-1383.

Tessier-Lavigne M (1994) Axon guidance by diffusible repellants and attractants. Curr Opin Genet Dev 4:596-601.

Vactor DV, Sink H, Fambrough D, Tsoo R, Goodman CS (1993) Genes that control neuromuscular specificity in Drosophila. Cell 73:1137-1153.

Vielmetter J, Kayyem JF, Roman JM, Dreyer WJ (1994) Neogenin, an avian cell surface protein expressed during terminal neuronal differentiation, is closely related to the human tumor suppressor molecule deleted in colorectal cancer. J Cell Biol 127:2009-2020.

Wright JW, Snyder MA, Schwinof KM, Combes S, Copenhaver PF (1999) A role for fasciclin II in the guidance of neuronal migration. Development 126:3217-3228.

Zhang YQ, Broadie K (1999) Cloning, mapping, and tissue-specific expression of Drosophila clathrin-associated protein AP50 gene. Gene 233:171-179. 